Reprod. Nutr. Dévelop. 1980, 20 (1 A), 23-59.

\title{
Polymorphisme d'origine géographique portant sur la « valence » des chromosomes sexuels et phénomènes de monogénie résultant du croisement de différentes populations de Porcellio dilatatus Brandt (Crustacé Isopode Terrestre)
}

\author{
par J.-J. LEGRAND, P. JUCHAULT, J.-P. MOCQUARD, G. MARTIN \\ Laboratoire de Physiologie et Génétique des Crustacés, \\ E.R.A. CNRS no 230 \\ 40, Avenue du Recfeur Pineau 86022 Poitiers cedex.
}

Summary. Geographic polymorphism of the « valence » of sexual chromosomes, and the monogeny resulting from the crossing of different populations of Porcellio dilatatus Brandt (Crustacea, Isopoda, Oniscoidea).

The studied population of Porcellio dilatatus dilatatus comes from Neuvy-en-Dunois (Eure). This subspecies is found throughout continental France. Samplings in the field always show a normal sex-ratio, but numerous females are intersexual. The intersexuality reported in this study (fertile females with external male characters) has been observed in other populations where it resulted from an epigenetic factor which is infectious, thermosensitive and transmitted by both sexes. In lineages obtained from intersexed females collected in Neuvy and reared in the laboratory, intersexuality quickly fated, but some cases of monogeny (one sex prevailing in a brood) appeared. The lineage of mainly one female ( $q \mathrm{Bi} \mathrm{IV}$, tabl. 1) was studied by inbreeding. These animals were also crossed with others belonging to outside populations, such as the Poitiers population in which the males are heterogametic and the St-Honorat Island (Alpes mar.) population belonging to another subspecies, $P$. $d$. petiti, in which the males are homogametic. Some crossings used physiological neo-males, i.e. females whose physiological sex had been inversed early by implantation of an androgenic gland. One of the $F_{2}$ generations ( $n^{\circ} 17$, fig. 1 ) and one back-cross $\left(\mathrm{CRF}_{3}\right)$ among the $O \mathrm{Bi}$ IV descendants were arrhenogenic ( $\mathrm{Ar}$ ), i.e. contained more males than females (about 3 males for 1 female in this study). This feature was also observed in further generations (fig. 2), but the arrhenogenic crosses were always accompanied by amphogenic ones (A) (normal sex-ratio). By inbreeding amphogenic offsprings, either stable pure amphogenic lineages ( $T s_{2}$ and $T s_{5}$, fig. 3 ) or those with some arrhenogenic crosses (figs $3,4,5$ ) were obtained. Rare thelygenic offsprings $(T)$, composed of only females, were observed. Some females from such a thelygenic brood $\left(F_{5} \mathrm{Bi} I V 10=17\right.$; ; fig. 4) were crossed with Poitiers males $(2 \mathrm{~A} X Y)$. The first generation was apparently amphogenic, but some arrhenogenic offsprings were again found in the $F_{2}$ generations. Hybrid males from these arrhenogenic broods were crossed with different females (table 2) : sisters (S), Poitiers females $(\mathrm{P})$, Neuvy females either from a stable amphogenic $\left(\mathrm{Ts}_{5}\right.$ ) or arrhenogenic $\left(A r_{4}, 1,2\right.$ and 3$)$ lineage or from thelygenic offspring $\left(\mathrm{Ar}_{4} \mathrm{~T}\right)$. These crosses showed at least two types of males and one type of female in the arrhenogenic lineages, whereas in amphogenic lineages, two types of females could be distinguished by their crossing with certain 
males $(\approx 3, \delta 4$; table 2$)$ : the first gave amphogenic offsprings and the second arrhenogenic ones. Hybrid males from arrhenogenic offspring gave either amphogenic or thelygenic offspring when they were crossed with Poitiers females ( $2 \mathrm{~A} X X)$. The foregoing results would indicate that there were three types of males in the arrhenogenic lineages. Neomales were obtained by experimental sex inversion of young females from pure Neuvy and hybrid strains. Their genetical study corroborated the heterogeneity of Neuvy females : some were homogametic $(2 \mathrm{AXX})$ and others were heterogametic $(2 \mathrm{AXZ})$. Crosses of the Poitiers and Neuvy strains showed the equivalence of the $X$-chromosomes from both origins; the $Y$-chromosomes were also comparable. The $X$-chromosome of either strain was always epistatic on the Z-chromosome. The lineage of the + Bi IV could be reconstituted, supposing that the starting cross was:

$$
\mathrm{F}_{\mathbf{1}}=\hat{\jmath} \mathrm{XY} \times q \mathrm{XZ} \rightarrow q \mathrm{XX}+q \mathrm{XZ}+\hat{\jmath} \mathrm{XY}+\hat{\jmath} \mathrm{YZ}
$$

In the $F_{2}$ generations, three types of amphogenic offsprings and one arrhenogenic one should be found :

$$
\begin{aligned}
& (A)_{1}=\hat{\jmath} X Y \times q X X \rightarrow \hat{X} X Y+q X X \\
& (A)_{2}=\hat{o} X Y \times q X Z \rightarrow \text { like } F_{1} \text { generation } \\
& (A)_{3}=\delta^{\star} Y Z \times q X X \rightarrow o^{*} X Y+q X Z \\
& (\mathrm{Ar})=\hat{\jmath} \mathrm{YZ} \times \phi \mathrm{QZZ} \rightarrow \hat{\jmath} \mathrm{XY}+\hat{\jmath} \mathrm{YZ}+\hat{\jmath} \mathrm{ZZ}+\phi \mathrm{XZ} \text {. }
\end{aligned}
$$

The $Z Z$ males from Ar offspring yielded two new types of offsprings: amphogenic,

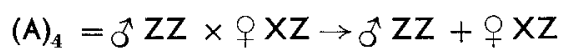

and thelygenic,

$$
\text { (T) }=\delta \mathbf{Z Z} \times q \mathbf{X X \rightarrow} \rightarrow \mathbf{X Z} \text {. }
$$

By inbreeding, the broods of $(A)_{1}$ and $(A)_{4}$ types led to stable amphogenic strains, while arrhenogeny would appear in the lineages from the $(A)_{2}$ and $(A)_{3}$ types. Crossings between siblings of arrhenogenic offspring produced one arrhenogenic for two amphogenic offsprings. This theoretical pattern must be completed by additional observations. Rare males might appear in crosses where genetical equation required pure thelygeny (III.4a). These males were demonstrated to be spontaneously inversed $X Z$ females. This raised the question of the identity of the masculinizing factor responsible for sex inversion and that inducing female intersexuality. More frequently, $\mathrm{ZZ}_{0}{ }^{\lambda}$ became neo-females and yielded two types of monogeny, one a strong genetical arrhenogeny perpetuated by the presence of neofemales, and the other a partial thelygeny (40 p. 100 of males) which was transmitted and could be selected (III.6b). The nature of the feminizing factor involved has been discussed. The sex-ratio varied significantly in the successive broods of some females. This phenomenon (allelogeny) might be explained by the epigenetic nature of the different factors inversing sex. Neuvy strain animals and Neuvy $\times$ Poitiers hybrids were crossed with indi-

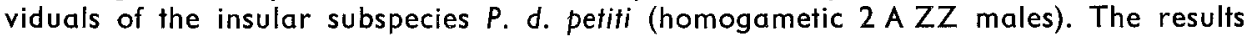
confirmed the coexistence of homo- and heterogametic females in the Neuvy population, and showed that the Z-chromosome was equivalent in both populations. The crosses between insular males and Poitiers females were practically infertile; this seemed to result from the absence of symbiotic bacteroids in the ovaries of the latter, which were present in the insular females. The same infertility was observed in the insular $\hat{\delta} \times$ Neuvy $O$ cross ; the females of that population lacked symbiotic bacteroids. Thus, this symbiont did not appear to be related to female heterogamy and was different from that of Armadillidium vulgare which inverses the genetical males into neo-females (Martin ef al., 1973). The Neuvy population of $P$. $d$. dilatatus probably arose from the hybridization between a heterogametic male strain and a homogametic male strain. The first was closely related to that of Poitiers, and the second was not very different from the $P$. $d$. pefiti strain of Saint-Honorat Island. The $X$-chromosome valence was the same in these various lineages, but the $Y$-chromosome one varied (hypostatic $Z$ state as compared to $X$ in heterogametic female strains). These phenomena have been compared with analogous studies on fishes. 
Matériel et méthodes.

Terminologie employée.

I. - $F_{1}$ des femelles infersexuées.

II. - Filiations réalisées en inbreeding à partir de la $ᄋ$ $\mathrm{Bi}$ IV.

III. - Filiations des croisements entre descendances Bi IV de Neuvy et population de Poitiers.

IV. - Emploi de néo-mâles expérimentaux du type Neuvy dans divers croisements.

V. - Comparaison des valences des déterminants sexuels des populations de Neuvy et de Poitiers.

VI. - Reconstitution théorique et expérimentale des filiations Bi IV observées en inbreeding et outbreeding.

VII. - Filiation des croisements entre descendances Bi IV de Neuvy ef population insulaire de Saint-Honorat de $P$. dilatatus petiti.

VIII. - Données concernant d'autres individus de la population de Neuvy.

IX. - Conclusion générale.

\section{Introduction.}

Les données actuelles concernant la génétique du sexe de l'Oniscoïde Porcellio dilatafus peuvent se résumer comme suit. Il a été montré que la population de Poitiers, correspondant à l'espèce type, $P$. $d$. dilatatus, offre une homogamétie femelle (Juchault et Legrand, 1964), contrastant avec celle de $P$. $d$. petiti de l'île Saint-Honorat (AlpesMaritimes), à hétérogamétie femelle (Legrand ef al., 1974), résultat confirmé par l'étude de leur hybridation au laboratoire (Legrand ef Juchault, 1974).

Legrand et al. (sous-presse) ont pu rapporter l'avortement des croisements : ô petiti $\times$ q dilatalus à l'absence d'un bactéroïde symbiote dans les ovocytes de l'espèce type, alors que la sous-espèce insulaire en est pourvue.

La monogénie est pratiquement inconnue chez cette espèce, à part une arrhénogénie non transmissible résultant du croisement de mâles normaux avec des néofemelles spontanées, généralement intersexuées ( $q$ à apophyse génitale mâle), observées dans plusieurs populations continentales et rapportées à l'action d'un facteur féminisant labile qui imprime à la différenciation sexuelle un décours hermaphrodite protandrique (Legrand et Juchault, 1972b).

Trois autres types d'intersexualité ont été décrits, sans lien avec des phénomènes de monogénie :

- mâles à ouvertures génitales femelles ( $\widehat{o} o g)$, présents dans plusieurs populations : Corbeil (Legrand J. J. el Legrand H., 1947), Chinon, Roscoff, Saint-Honorat (résultats inédits) ;

- femelles fonctionnelles différenciant précocement (et souvent asymétriquement) des caractères sexuels externes de type mâle: gonopodes ef péréiopodes; ce type d'intersexualité, peu fréquent, a été précisément décrit à partir de la population de Neuvy-en-Dunois (Legrand, 1956), retrouvé dans celle de Rom (Deux-Sèvres) (Juchault, 1966 ; Juchault el Legrand, 1970, 1975) et a été qualifié de pseudo-hermaphrodisme masculin externe de «type $A$ », par opposition à un «type B » d'intersexualité présent dans de nombreuses populations et caractérisé par la différenciation aible et fardive de caractères sexuels externes mâles limités aux gonopodes. 
Nous avons montré que le pseudohermaphrodisme de type $A$ est dô à un facteur épigénétique infectieux et polytrope (virus ?) thermosensible, susceptible d'être transmis à la descendance à la fois par voie matrocline et patrocline.

L'étude suivante a été entreprise pour voir s'il existait dans la population de Neuvy-en-Dunois une corrélation entre mécanisme de détermination du sexe et état intersexué de nombreux individus. Des phénomènes de monogénie répondant à un mécanisme jusqu'ici inconnu dans la nature ont justifié la poursuite de l'étude, malgré la disparition rapide, en élevage, du pseudo-hermaphrodisme de type A.

\section{Matériel et méthodes.}

La population de Porcellio dilatatus qui fait l'objet essentiel de cette étude provient d'une cave profonde située à Neuvy-en-Dunois (Eure), ef servant au stockage de denrées alimentaires, notamment de pommes de terre. Morphologiquement, elle appartient à la sous-espèce type : $P$. dilatatus dilatatus, qui peuple la majeure partie de la France (Vandel, 1962). Trois échantillons ont été récoltés dans cette station, à quelques années d'intervalle $(1955,1956,1960)$. Ils sont caractérisés, d'une part, par une proportion des sexes non significativement différente de la normale (exemple : 48 今. 58 우 en 1956), d'autre part, par une forte proportion (22/48 en 1956) de femelles intersexuées correspondant au pseudohermaphrodisme masculin externe de type $A$.

A partir de la récolte de 1955, des femelles intersexuées pubères ont été mises en élevage, en salles climatisées (température de $20^{\circ} \mathrm{C} \pm 1$, photopériode naturelle), sans réaccouplement, et leurs descendances étudiées - pour certaines jusqu'à la $F_{15}$ - les unes en inbreeding, d'autres en outbreeding, d'une part, avec une population poitevine de la même sous espèce, d'autre part, avec une population de P. d. petiti originaire de l'île Saint-Honorat (Alpes-Maritimes) (Legrand et al., 1976).

En l'absence de mutations liées au sexe - encore inconnues chez les Oniscoïdes - les phénomènes de monogénie ont été étudiés en analysant les proportions des sexes de chaque descendance et en les comparant - à l'aide du $\chi^{2}$ - aux prévisions fondées sur les équations hétérosomiques classiques.

Pour aider à la compréhension des phénomènes génétiques, des néomâles physiologiques ont été créés par implantation de glandes androgènes chez de très jeunes femelles de différentes lignées et employés dans divers croisements.

\section{Terminologie employée.}

Notion de «valence » des déterminants sexuels. - La notion de « valence » a été introduite par Goldschmidt (1938) pour comparer quantitativement les facteurs $M$ et $\mathrm{F}$ de différentes races géographiques de Lymantria dispar et rendre compte de l'apparition d'intersexués diploïdes dans certains croisements. Lorsque la différence entre ces valences afteint un seuil, ou "minimum épistatique », intervient un développement normal en mâle ou en femelle ; en-dessous de ce seuil, il y a apparition d'intersexués. Dans le même ordre d'idée considérant le sexe comme la résultante d'une balance génique, différents auteurs, tels Kosswig, de Lattin (références in Bacci, 1965) ont utilisé la notion d'épistasie pour définir la prévalence des gènes polymériques de 
l'un des sexes sur la somme des gènes du sexe opposé. Partant ici de la conception que les hétérochromosomes portent les déterminants sexuels majeurs (cf. conclusion générale) et que le sexe des hybrides nés du croisement de 2 lignées est ainsi le plus souvent l'expression de leur constitution hétérosomique, on employera le terme de "valence » pour traduire simplement le fait que, chez un mâle hétérogamétique de type $X Y$, le-ou les gènes sexuels mâles contenus dans $Y$ surpassent le(ou les) gènes(s) femelle(s) contenu(s) dans $X$, alors que c'est l'inverse dans le cas de l'hétérogamétie femelle, où le (ou les) gènes femelles de $X$ sont épistatiques sur le(ou les) gène(s) mâle(s) de $Z$. Ceci sera repris dans la conclusion générale.

\section{I. - $F_{1}$ des femelles intersexuées}

8 femelles intersexuées pubères (et fécondées dans la nature par des mâles inconnus) ont donné, en 2 ans, 1 à 3 portées dont le détail est analysé dans le tableau 1 . Au total, l'ensemble des $F_{1}$ est homogène $\left(\chi^{2}=8,47 \mathrm{DL}=7\right)$ et amphogène (219 $\hat{\sigma} \delta$, 204 웅). Néanmoins, l'une d'elles (Bi $\mathrm{VI})$ offre une descendance à tendance arrhénogène $\left(\chi^{2}=5,06\right)$ dont la signification sera discutée en fonction de données ultérieures (chapitre VIII). II est à souligner que l'ensemble des $F_{1}$ offre généralement une forte proportion de femelles intersexuées, sauf lorsque la génitrice est faiblement intersexuée.

\section{TABLEAU 1}

$F_{1}$ des femelles infersexuées récoltées dans la nature à Neuvy-en-Dunois $(i=q$ intersexuée type $A$ et $B$ : cf. texte)

No génitrice

Degré d'intersexualité

$\frac{\text { Descendants }\left(F_{1}\right)}{3 \sigma^{*}} \frac{\phi Q}{\text { total i de type A }}$

\begin{tabular}{|c|c|c|c|c|}
\hline I & très fort (type A) ............... & 28 & 32 & 19 \\
\hline II & très faible (type B) ........ & 12 & 9 & 0 \\
\hline III & faible (type A) & 54 & 63 & 0 \\
\hline IV & très fort (type A) ................ & 38 & 39 & 5 \\
\hline V & fort (type A) $\ldots \ldots \ldots \ldots \ldots \ldots \ldots$ & 11 & 16 & 8 \\
\hline VI & fort (type A) & 41 & 23 & 2 \\
\hline VII & moyen (type A) & 12 & 8 & 4 \\
\hline VIII & fort (type A) & 13 & 16 & 10 \\
\hline
\end{tabular}

\section{II. - Filiations réalisées en inbreeding à partir de la $q$ Bi IV}

A partir des 4 portées amphogènes constituant la $F_{1}$ de la $q \mathrm{Bi} I V$, sur $28 \mathrm{~F}_{2}$ effectuées, 16 seulement ont été fécondes. L'une d'elles (n $\left.n^{\circ} 17\right)$ est arrhénogène : $35 \widehat{\delta} \widehat{\delta}, 8$ 우 ; les autres constituent un ensemble homogène $\left(\chi^{2}=13,92-D L=14\right)$ 
comportant $136 \widehat{\delta} \widehat{\delta}, 120$ 우오. En fait, pour beaucoup, le nombre trop restreint de descendants n'a pas permis de mettre en évidence des sex-ratios significativement différentes de la normale. Les filiations de 6 des $F_{2}$ obtenues ont été étudiées, selon le cas, jusqu'à la $F_{5}, F_{7}$ ou $F_{8}$ ef résumées dans les figures 1 à 5 (pour la commodité de cette présentation, certaines descendances statistiquement homogènes - amphogènes pour la plupart - ont dû être groupées).

\section{- Résultats.}

1. L'arrhénogénie observée dans la $F_{2} n^{0} 17$ se retrouve en $F_{3} 17 \mathrm{~A}$ et en $C_{R} F_{3} 6 \mathrm{~A}$, les autres $F_{3}$ obtenues étant amphogènes (fig. 1). L'éfude des filiations issues du $C R F_{3}$ $6 \mathrm{~A}$ (fig. 2) montre une transmission de l'arrhénogénie en $F_{4}$, puis - très généralement - une disjonction, à chaque génération, conduisant à l'apparition - à partir

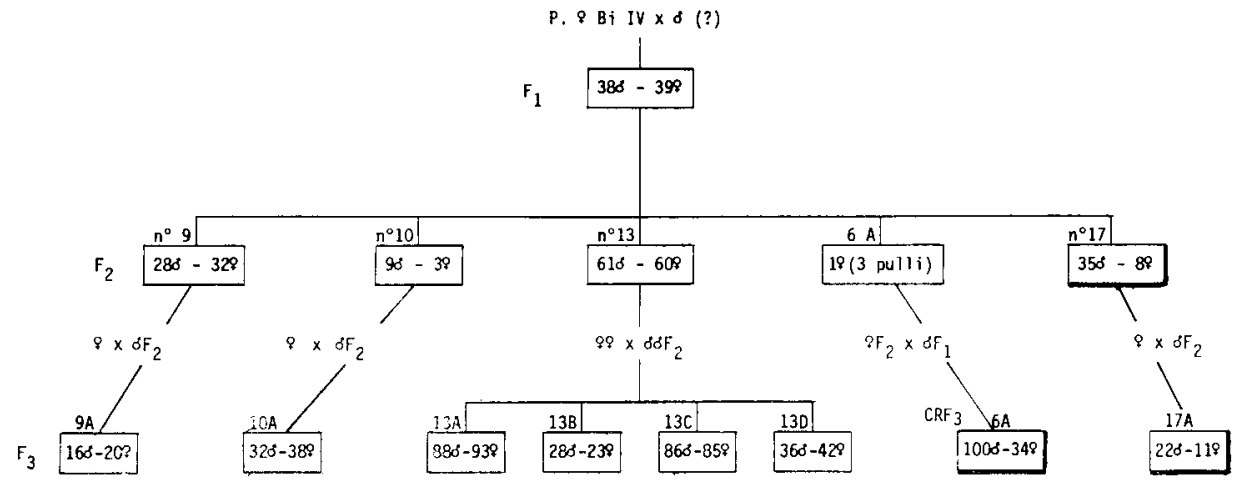

FIG. 1. - Généalogie $\left(F_{1}\right.$ à $\left.F_{3}\right)$ de la femelle $B i$ IV. Les descendances arrhénogènes sont inscrites dans un cadre renforcé, les amphogènes dans un cadre simple.

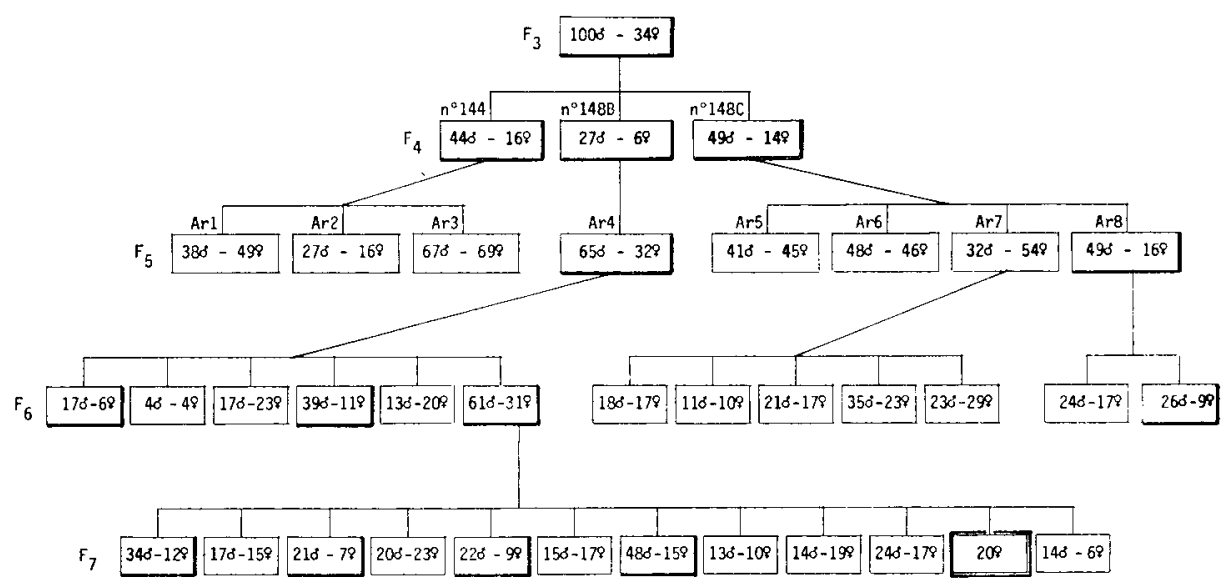

FIG. 2. - Généalogie $\left(F_{3}\right.$ ò $\left.F_{7}\right)$ de la descendance arrhénogène $\mathrm{CRF}_{3} 6 \mathrm{~A}$.

Même représentation que précédemment; la descendance thélygène est soulignée d'un double trait. 


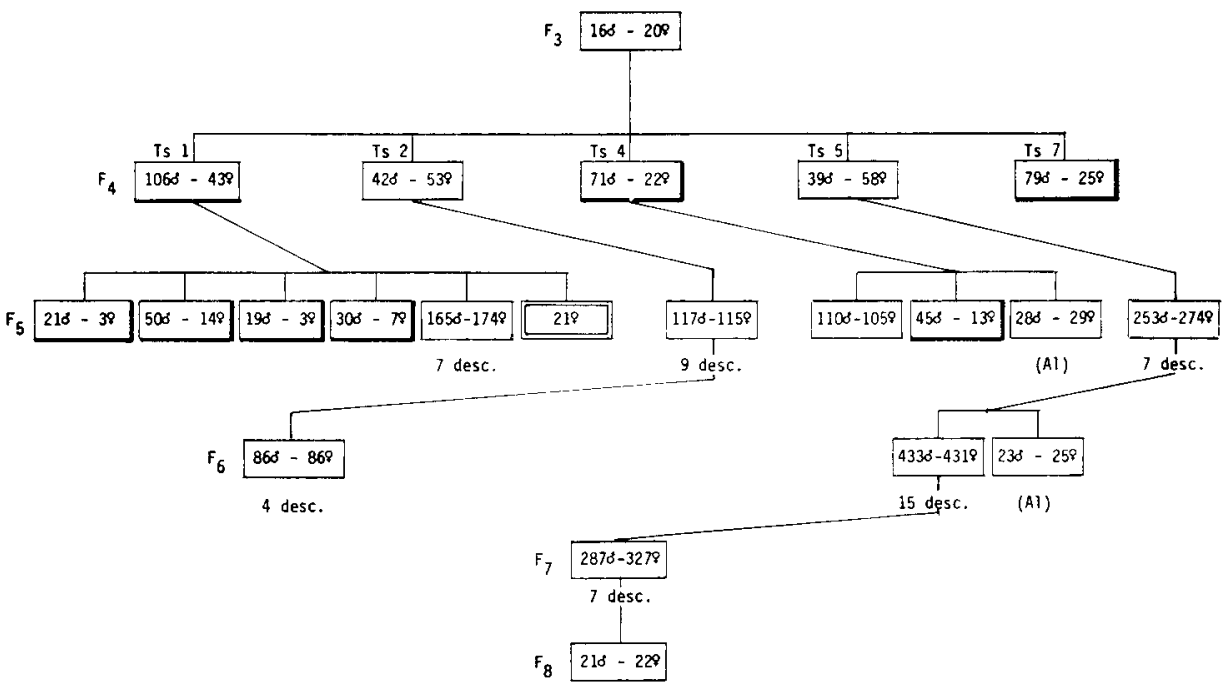

FIG. 3. - Généalogie $\left(F_{3}\right.$ à $\left.F_{7}\right)$ de la descendance amphogène $F_{3} 9 A$. Certaines descendances, homogènes, ont été regroupées ef leur nombre indiqué sous le cadre. $(\mathrm{Al})$ : descendances allélogènes (voir texte).

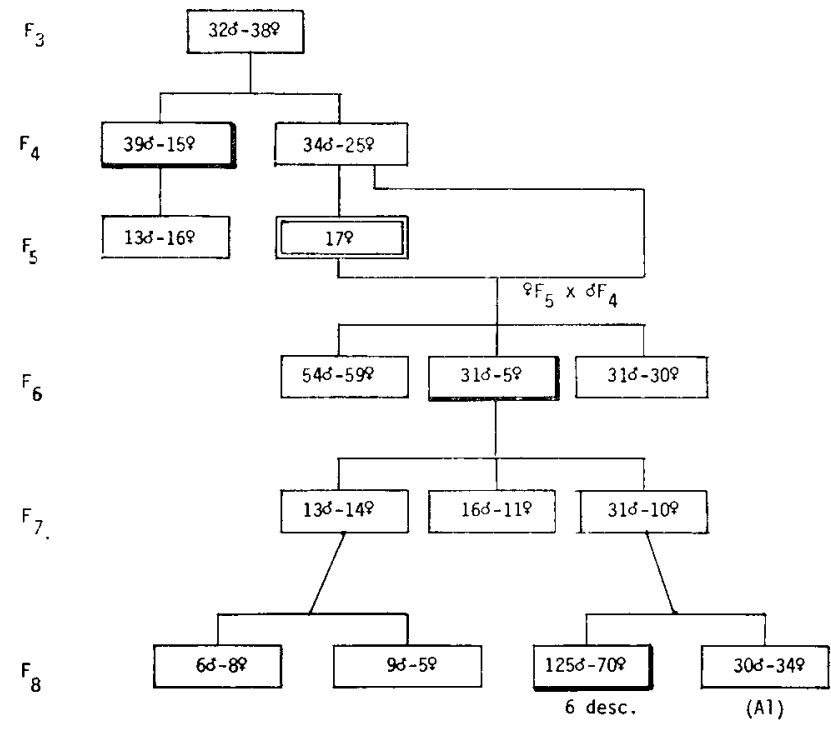

FIG. 4. - Généalogie $\left(F_{3} a ̀ F_{8}\right)$ de la descendance amphogène $F_{3} 10 A$. Mêmes conventions que précédemment. 


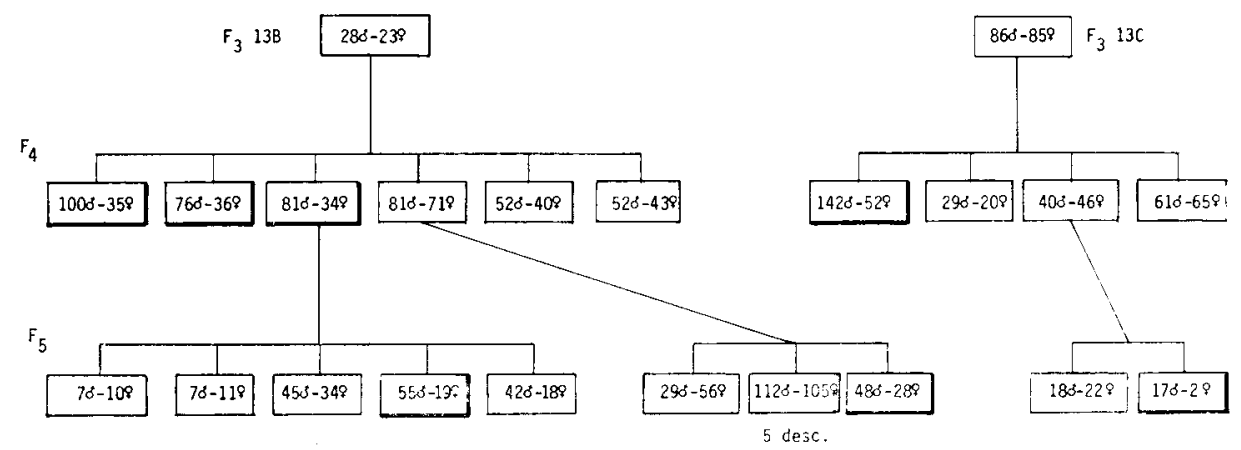

FIG. 5. - Généalogie $\left(F_{3}\right.$ ò $\left.F_{5}\right)$ des descendances amphogènes 13B ef 13C. Mêmes conventions que précédemment.

d'une descendance arrhénogène - de descendances amphogènes et arrhénogènes : néanmoins, en $F_{7}$, apparaît une descendance thélygène pure. Les descendances arrhénogènes obtenues constituent un ensemble homogène (602 $\hat{0} \hat{o}, 218 \hat{\phi}$ 우) $\left(\chi^{2}=7,40-\mathrm{DL}=13\right)$ ne différant pas statistiquement du rapport $3 \delta \delta / 1 q$ $\left(\chi^{2}=1,09\right)$. De même, l'ensemble des amphogènes est homogène $\left(\chi^{2}=23,25-\right.$ $\mathrm{DL}=21)$.

2. A partir des $\mathrm{F}_{3}$ amphogènes $(9 \mathrm{~A}, 10 \mathrm{~A}$ et $13 \mathrm{~A}$ ), on observe également une disjonction en descendances amphogènes ef arrhénogènes qui redonnent, les unes ef les autres, ce même type de disjonction, comportant en outre de rares thélygènes et, de plus, des descendances allélogènes. Ainsi, en $F_{5} T_{4}$ (filiation $9 \mathrm{~A}$ ), la descendance globalement amphogène $28 \hat{\sigma} \hat{\sigma}, 29 q q$ correspond d̀ 2 portées hétérogènes :

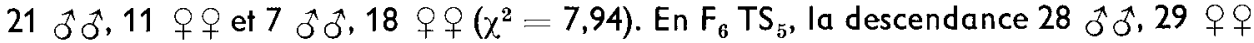
rassemble 3 portées hétérogènes : $3 \hat{\jmath} \hat{\jmath}-9 \hat{\jmath} \hat{\sigma}, 18$ 우 $-11 \hat{\jmath} \hat{\sigma}, 7 q$ 우 $\left(\chi^{2}=6,82-D L=2\right)$ (fig. $\left.3,4,5\right)$.

De même, en $F_{7} 10 \mathrm{~A}$, la descendance $30 \hat{\partial} \hat{o}, 34 q 9$ groupe 4 portées hétéro-

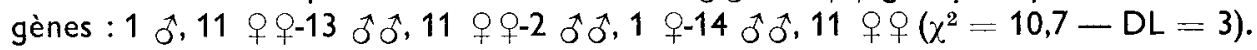
On doit néanmoins souligner qu'une amphogénie stable a pu être sélectionnée à partir des descendances $\mathrm{TS}_{2}$ ef $\mathrm{TS}_{5}$ issues de la $\mathrm{F}_{3} 9 \mathrm{~A}$ (fig. 3). En effet, outre la permanence de l'amphogénie dans 14 descendances portant sur 3 générations en $\mathrm{TS}_{2}$, et 31 descendances portant sur 5 générations en $\mathrm{TS}_{5}$, un terrarium a été constitué depuis juin 1971, rassemblant les mâles ef les femelles des générations issues de la $F_{5} \quad \mathrm{TS}_{5}$. 33 descendances analysées à partir de femelles isolées gravides de ce terrarium constifuent un ensemble amphogène et homogène (1 $053 \hat{\jmath} \hat{\sigma}, 1090 q$ q $\left.-\chi^{2}=27,42-D L=32\right)$. Cependant, une de ces descendances globalement amphogène, est allélogène (1 re portée : $18 \hat{\delta} \widehat{\delta}, 11$ q $Q$; $2^{\mathrm{e}}$ por-

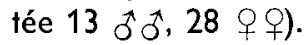

Enfin, 6 croisements effectués à partir d'une des descendances amphogènes ont abouti à de l'amphogénie (ensemble homogène constitué par $134 \delta \hat{\delta}$ et 133 우, $\chi^{2}=3,74-\mathrm{DL}=5$ ). 
Si l'on s'en tient au type de disjonction offert par le croisement d'individus nés dans une descendance arrhénogène, en réunissant les données apportées par l'inbreeding on constate que sur 60 croisements : 19 ont donné des descendances (Ar) et 41 des descendances non (Ar), essentiellement amphogènes.

A ce stade de l'analyse, il s'avère que l'arrhénogénie observée ne peut être expliquée par un modèle comparable à celui rapporté dans l'introduction, c'est-àdire le croisement d'un ổ normal hétérogamétique $(2 \mathrm{~A} X Y)$ avec une néo- + de même génotype. En effet, si, dans ce cas, la $F_{1}$ aurait également été arrhénogène du type $3 \hat{\delta} \delta / 1$ q, à la génération suivante, on aurait dû observer des descendances arrhénogènes pures, dues aux mâles $2 A Y Y$ et des amphogènes (dues aux mâles $2 \mathrm{~A} X Y$ ), dans le rapport 1 (Ar)/2 (A), ce qui n'est pas. Il est à noter que l'intersexualité de type $A$, encore présente chez 2 des 128 femelles obtenues en $F_{2}$, a totalement disparu dans les générations ultérieures. Une intersexualité de type $B$ est apparue chez 13 femelles $F_{2}$ : 2 provenaient de génitrices de type $A, 11$ de génitrices normales. Ce type d'intersexualité n'a pas été recherché dans les générations ultérieures, étanł donné son apparition très tardive.

\section{III. - Filiations des croisements entre descendances Bi IV de Neuvy et population de Poitiers}

Ces croisements ont été entrepris pour analyser les conséquences possibles, sur la sex-ratio des descendances Bi IV, de l'introduction de mâles reconnus comme étant hétérogamétiques et appartenant à une lignée amphogène marquée par le gène autosomique récessif fulvum (Legrand, 1962). La descendance Bi IV utilisée pour ces croisements en outbreeding est la $F_{5} B i$ IV 10 thélygène (17 $q$ q $)$.

1. Deux croisements $F_{1}$ : $\hat{o}$ (lignée poitevine) $\times \not F_{5} B i$ IV 10 , ont abouti à des

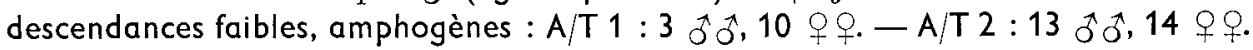
2a. $4 \mathrm{~F}_{2}$ ont été effectuées à partir de $A / T 1: 3$ des descendances obtenues sont ampho-

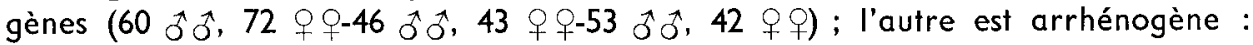

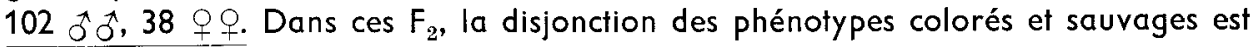
normale, avec $1 / 4$ de fulvum répartis d'une façon égale dans les 2 sexes. Le gène fulvum n'ayant pas d'incidence sur la sex-ratio, il n'en sera plus question dans les résultats.

2b. $6 \mathrm{~F}_{2}$ ont été effectuées à partir de $\mathrm{A} / \mathrm{T} 2: 4$ ont été amphogènes (au total, 148 $\widehat{\partial} \widehat{\delta}$,

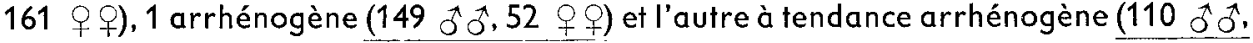
81 Q $q)$. Il y a donc réapparition, en $F_{2}$, de descendances, les unes amphogènes, d'autres arrhénogènes du type $3 \hat{\delta} \delta / 1$. Toutes les filiations ultérieures ont été obtenues à partir des descendances arrhénogènes du type $3 \hat{o} \hat{o} / 1$ q de $F_{2} A / T 1$ et $F_{2} A / T 2$.

3a. 6 mâles de ces descendances ont élé croisés en $F_{3}$, chacun avec 12 femelles : 3 우의 scurs, 3 q 9 de la lignée $B i$ IV pure, prises en $F_{5} A_{4}$ arrhénogène, 3 우 de la même lignée, prises en $\mathrm{F}_{5} \mathrm{TS}_{5}$ amphogène, enfin 3 q $q$ de la lignée poitevine amphogène. De plus, après 2 ans de reproduction avec les femelles précédentes, les 5 mâles survivants ( $\left.n^{\circ} 1,2,3,5,6\right)$ ont été croisés avec 1 à 2 q $q$ issues de la $F_{7} A R_{4}$ thélygène pure $(20 q q)$ (cf. fig. 2). 
- Résultats (tabl. 2).

Sur les 6 mâles provenant des descendances arrhénogènes hybrides, 4 (no 1 , $2,5,6)$ ont donné avec toutes les femelles des descendances amphogènes et homo-

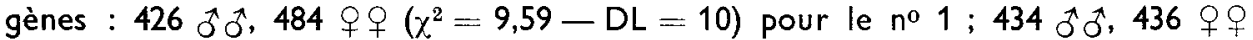
$\left(\chi^{2}=11,38-D L=10\right)$ pour le $n^{0} 2 ; 473 \delta \pi, 456$ 우 $\left(\chi^{2}=9,23-D L=11\right)$ pour le no $4 ; 704 \delta \hat{\delta}, 753$ q $n^{\circ}\left(\chi^{2}=16,43-D L=12\right.$ ) pour le $n^{0} 5$ (au total, $2037 \hat{\delta} \hat{\delta}, 2139$ 우). Les 2 autres mâles ont donné avec leurs sœurs, ainsi qu'arec toute femelle de Neury issue d'une descendance arrhénogène ou thélygène, des descendances arrhénogènes, dont l'ensemble est homogène et non significativement différent du type 3 ô ô/1 $q$ : (au total, 458 ô $\hat{o}, 165$ q $q\left(\chi^{2}=3,36-\mathrm{DL}=4\right)$

\section{TABLEAU 2}

Filiation des croisements entre descendances Bi IV Neuvy ef population de Poitiers. Origine des $\hat{\sigma}$ et des $Q$ : voir texte. $S=q$ sceurs des $\delta ; P=q$ de Poitiers. (Du fait que les résultats n'ont pas été encadrés).

\begin{tabular}{|c|c|c|c|c|c|c|}
\hline \multirow{2}{*}{ q } & \multicolumn{6}{|c|}{0} \\
\hline & 1 & 2 & 3 & 4 & 5 & 6 \\
\hline$S_{1}$ & $\begin{array}{l}79 \stackrel{\circ}{\circ} \\
74 \text { 古 }\end{array}$ & $\begin{array}{l}51 \stackrel{0}{\circ} \\
38 \stackrel{9}{q}\end{array}$ & $\begin{array}{r}148 f \\
44 \bigcirc\end{array}$ & $\begin{array}{l}90 \AA \\
21 \%\end{array}$ & $\begin{array}{l}68 \stackrel{1}{1} \\
66 \stackrel{9}{9}\end{array}$ & $\begin{array}{l}18 \AA \\
26 \stackrel{+}{+}\end{array}$ \\
\hline $\mathrm{S}_{2}$ & & $\begin{array}{l}71 \stackrel{*}{\circ} \\
83 \stackrel{9}{q}\end{array}$ & $\begin{array}{r}106 \circ \\
47 @\end{array}$ & $\begin{array}{r}105 \stackrel{0}{\circ} \\
42 \stackrel{q}{q}\end{array}$ & $\begin{array}{l}8 \hat{0} \\
8 \%\end{array}$ & $\begin{array}{r}105 \hat{\sigma} \\
889\end{array}$ \\
\hline $\mathrm{S}_{3}$ & $\begin{array}{l}44 \sigma^{*} \\
36 ?+\end{array}$ & $\begin{array}{l}160 \\
13 \%\end{array}$ & $\begin{array}{r}110 \% \\
44 \text { o }\end{array}$ & $\begin{array}{l}1370^{*} \\
420^{\circ}\end{array}$ & $\begin{array}{l}610 \\
62 O\end{array}$ & 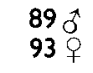 \\
\hline $\begin{array}{l}\mathrm{Ar}_{4} \\
1\end{array}$ & $\begin{array}{l}29 \AA \\
37 \stackrel{+}{q}\end{array}$ & $\begin{array}{l}290^{\circ} \\
23 \circ+\end{array}$ & $\begin{array}{l}67 \AA \\
21 \stackrel{9}{9}\end{array}$ & & $\begin{array}{l}23 \hat{\circ} \\
21 \stackrel{9}{9}\end{array}$ & $\begin{array}{l}21 \% \\
26 \%\end{array}$ \\
\hline $\begin{array}{l}\mathrm{Ar}_{4} \\
2\end{array}$ & & $\begin{array}{l}290^{\circ} \\
41 \stackrel{+}{+}\end{array}$ & & $\begin{array}{l}690^{\circ} \\
23 \text { 市 }\end{array}$ & $\begin{array}{l}40 \hat{\circ} \\
63 \stackrel{9}{9}\end{array}$ & $\begin{array}{l}11 \% \\
27 \stackrel{\circ}{0}\end{array}$ \\
\hline $\begin{array}{l}\mathrm{Ar}_{4} \\
3\end{array}$ & $\begin{array}{l}130 \\
130 \\
9\end{array}$ & $\begin{array}{l}47 \hat{\circ} \\
49 \stackrel{+}{+}\end{array}$ & & & & $\begin{array}{l}68 \hat{\circ} \\
62 \hat{+}\end{array}$ \\
\hline $\begin{array}{l}T_{1} S_{5} \\
\text {. }\end{array}$ & $\begin{array}{l}32 \% \\
28 \%\end{array}$ & $\begin{array}{l}21 \stackrel{*}{\circ} \\
14 \stackrel{+}{+}\end{array}$ & $\begin{array}{l}560 \\
67 \stackrel{9}{+}\end{array}$ & 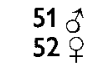 & $\begin{array}{l}30 \AA \\
28 \stackrel{+}{+}\end{array}$ & $\begin{array}{l}93 \text { न } \\
92 \text { 古 }\end{array}$ \\
\hline $\begin{array}{l}\mathrm{TS}_{5} \\
2\end{array}$ & $\begin{array}{r}73 \circ \\
102 \%\end{array}$ & $\begin{array}{l}52 o^{*} \\
61 \stackrel{+}{+}\end{array}$ & $\begin{array}{l}77 \stackrel{0}{\circ} \\
60 \stackrel{+}{q}\end{array}$ & $\begin{array}{l}63 \hat{\circ} \\
28 \circ\end{array}$ & $\begin{array}{l}65 \hat{\sigma} \\
54 \stackrel{+}{+}\end{array}$ & $\begin{array}{r}980 \\
117 \%\end{array}$ \\
\hline $\begin{array}{l}\mathrm{TS}_{5} \\
3\end{array}$ & $\begin{array}{l}60 \hat{\circ} \\
89 \stackrel{9}{f}\end{array}$ & $\begin{array}{l}700 \\
76 \%\end{array}$ & $\begin{array}{l}730 \\
66 \stackrel{0}{9}\end{array}$ & $\begin{array}{r}111 \stackrel{0}{\circ} \\
48 \stackrel{9}{9}\end{array}$ & $\begin{array}{l}60 \hat{\circ} \\
63 \stackrel{9}{+}\end{array}$ & $\begin{array}{l}61 \hat{0} \\
65 \stackrel{+}{0}\end{array}$ \\
\hline $\begin{array}{l}P \\
1\end{array}$ & $\begin{array}{l}10 \hat{\circ} \\
12 \stackrel{9}{+}\end{array}$ & $\begin{array}{l}29 \hat{\circ} \\
19 \stackrel{+}{+}\end{array}$ & $\begin{array}{l}29 \hat{\circ} \\
24 \hat{q}\end{array}$ & $\begin{array}{l}23 \stackrel{*}{\circ} \\
14 \stackrel{+}{+}\end{array}$ & $\begin{array}{l}29 \stackrel{0}{\circ} \\
31 \stackrel{+}{q}\end{array}$ & $\begin{array}{l}43 \text { o } \\
60 \stackrel{+}{9}\end{array}$ \\
\hline $\begin{array}{l}P \\
2\end{array}$ & $\begin{array}{l}40 \\
4 \stackrel{0}{+}\end{array}$ & $\begin{array}{l}33 \AA \\
24 \stackrel{9}{9}\end{array}$ & $\begin{array}{l}730 \\
580 \\
0\end{array}$ & $\begin{array}{l}980^{*} \\
96 \%\end{array}$ & $\begin{array}{l}17 \hat{0} \\
11 \stackrel{0}{9}\end{array}$ & $\begin{array}{l}410 \\
30 \text { 우 }\end{array}$ \\
\hline $\begin{array}{l}P \\
3\end{array}$ & $\begin{array}{l}55 x \\
629\end{array}$ & & $\begin{array}{l}480 \\
55 \text { ㅇ }\end{array}$ & $\begin{array}{r}22{ }^{\circ} \\
7 \stackrel{+}{q}\end{array}$ & $\begin{array}{l}42 \sigma^{*} \\
32 \%\end{array}$ & $\begin{array}{l}90 \\
90 \\
9\end{array}$ \\
\hline${ }_{T}^{\mathrm{Ar}_{4}}$ & $\begin{array}{l}27 \hat{\circ} \\
27 \hat{q}\end{array}$ & $\begin{array}{l}15 \% \\
18 \%\end{array}$ & $\begin{array}{r}270 \\
90\end{array}$ & & $\begin{array}{l}30 \hat{\sigma} \\
27 \stackrel{+}{q}\end{array}$ & $\begin{array}{l}47 \% \\
58 \text { क }\end{array}$ \\
\hline
\end{tabular}


pour le no 3 et $401 \delta \hat{\delta}, 128$ 우 $\left(\chi^{2}=3,3-D L=3\right)$ pour le $n^{\circ} 4$; au total : $859 \hat{\delta} \delta$. 293 우우 $\left(\chi^{2}\right.$ versus $3 \hat{\delta} \hat{\delta} / 1$ 웅 0,11$)$. Par contre, avec la plupart des femelles amphogènes : Neury du type $\mathrm{TS}_{5}$, d'une part, et poitevines, d'autre part, ces 2 mâles ont offert des descendances amphogènes, exception faite pour le of no 4 avec 2 femelles $\mathrm{TS}_{5}$ sur 3 et une poitevine sur 3 , où les descendances ont été arrhénogènes et non significativement différentes du type $3 \stackrel{\delta}{\delta / 1} q$.

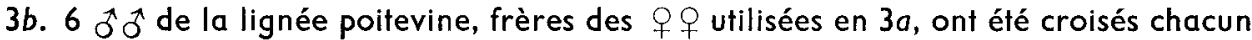
avec 3 우 $F_{2} A / T_{1}$ ou $A / T_{2}$, sœurs des $\widehat{\partial} \delta$ et des $q$ i également utilisés en $3 a$. Toutes les descendances ont été amphogènes ; l'ensemble, homogène représente : $1077 \hat{\sigma} \delta$, 1081 우우 $\left(\chi^{2}=12,3-\mathrm{DL}=16\right)$.

En conclusion provisoire, il apparaît qu'existent dans une descendance arrhénogène hybride du type $3 \hat{\sigma} \hat{d} / 1$ q, telle que $F_{2} A / T, 2$ types de mâles et un seul type de femelles; un 1er type de mâles, que l'on peut qualifier d'arrhénogène (Ar), est capable de redonner avec toute femelle sœur, hybride Neuvy-Poitiers, ou une $q$ du type Neuvy pur issue d'une descendance arrhénogène ou thélygène $\left(F_{5}\right.$ ou $\left.F_{7} A r_{4}\right)$, une descendance arrhénogène ; l'autre type de mâles, que l'on peut qualifier d'amphogène $(A)$ ef qui semble en nombre deux fois plus grand que le $1 \mathrm{er}$, donne une descendance amphogène. La conception de l'existence d'un seul type de femelle dans les descendances arrhénogènes est appuyée par l'uniformité des résultats des croisements de ces femelles avec des mâles de la lignée amphogène poitevine $(3 b)$. Par ailleurs, dans les lignées amphogènes : poitevine d'une part, $F_{5} T_{5}$ d'autre part, semblent exister 2 types de femelles que l'on peut définir d'après le résultat de leur croisement avec un $\widehat{\jmath}(\mathrm{Ar})$ : à savoir une descendance amphogène, ou une descendance arrhénogène.

4. Filiation des descendances arrhénogènes hybrides.

4a. Croisements : $ð(\mathrm{Ar}) \times q$ scur.

Des mâles nés dans les descendances arrhénogènes hybrides $\left(F_{3}\right.$ Neuvy-Poitiers) ont été croisés avec 1 à 3 de leurs sœurs. En répétant ce type de croisement sur 4 générations $\left(F_{4}\right.$ à $F_{7}$ de l'outbreeding), en tout, 180 croisements de ce type ont été effectués, dont les résultats peuvent être groupés en 8 ensembles d'après l'origine des géniteurs. Dans chaque ensemble :

a) Certains mâles ont donné avec toute les femelles auxquelles ils ont été croisés, une descendance arrhénogène, généralement du type $3 \delta \delta / 1$ 으, mais avec parfois un léger excès de femelles.

b) Les autres mâles, frères des précédents, ont donné :

- en très grande majorité des descendances amphogènes, avec parfois des oscillations de la sex-ratio, les unes vers une tendance thélygène (exemples : $70 \hat{\delta} \widehat{\partial}$. 140 우 ; $162 \hat{o} \hat{O}, 250$ 욱), les autres vers une tendance arrhénogène (exemple : 99 ふૈ

- de rares descendances allélogènes, exemples :

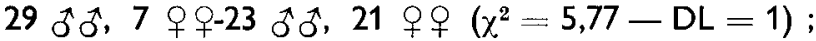

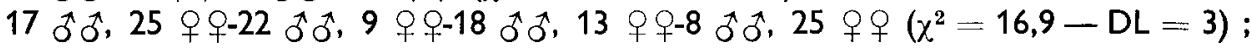

- enfin 2 descendances thélygènes : 51 $q q$ pour l'une, 173 우 $q$ ef 1 exceptionnel pour l'autre. 
Il $a$ été vérifié, en utilisant des mâles géniteurs de descendances arrhénogènes pour féconder, l'année suivante, des femelles ayant présenté au préalable une descendance amphogène avec un premier mâle, que la nouvelle descendance évoluait vers l'arrhénogénie après épuisement des spermatozoïdes du $1 \mathrm{er}$ mâle. On peut donc considérer que c'est bien le mâle qui est responsable de l'arrhénogénie.

Dans chacun des 8 ensembles, les disjonctions entre descendances arrhénogènes et non arrhénogènes - c'est-à-dire entre $\hat{o}(\mathrm{Ar})$ et non $(\mathrm{Ar})$ - sont comparables :

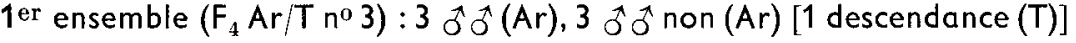

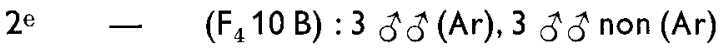

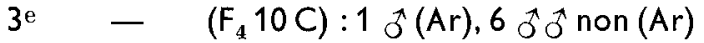

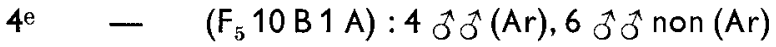

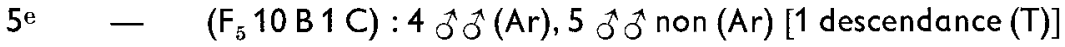

$$
\begin{aligned}
& \text { 6e - }\left(F_{6} 10 B 1 C\right): 3 \text { कै (Ar), } 2 \text { की non (Ar) } \\
& 7 \mathrm{e} \quad-\quad\left(F_{7} 10 \mathrm{~B} 1 \mathrm{C} 5-9\right): 2 \hat{O} \hat{\sigma}(\mathrm{Ar}), 8 \hat{O} \hat{O}^{\hat{O}} \text { non (Ar) } \\
& 8 \mathrm{e} \quad-\quad\left(\mathrm{F}_{7} 10 \mathrm{~B} 1 \mathrm{C} 5 \mathrm{~B}\right): 28 \mathrm{O}^{\hat{O}}\left(\mathrm{Ar}, \mathrm{O}^{4} 49 \mathrm{O}^{\hat{\alpha}}\right. \text { non (Ar). }
\end{aligned}
$$

Le test du $\chi^{2}$ donne une valeur de 5,06 pour $7 \mathrm{DL}$, montrant l'homogénéité de ces disjonctions. On peut ainsi considérer que, sur les 130 mâles, 48 sont (Ar) et 82 non (Ar), soit $1 \hat{o}$ (Ar) sur $3 \hat{o} \hat{o}$. Cette proportion n'est pas significativement différente des résultats obtenus en filiation arrhénogène dans le cas de l'inbreeding [13 $\hat{\sigma} \widehat{\delta}(\mathrm{Ar})$

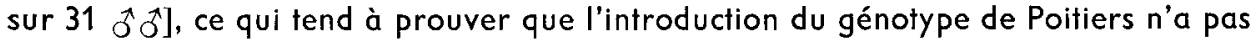
modifié le mécanisme d'apparition et de transmission de l'arrhénogénie existant dans la population de Neuvy.

4b. Croisements : $\widehat{O}(\mathrm{Ar}) \times$ क amphogène poitevine.

20 mâles issus d'une descendance $F_{7}$ hybride arrhénogène de type $3 \hat{\delta} \delta^{A} / 1$ 우 ont été croisés, chacun avec 4 femelles poitevines. Les résultats sont les suivants :

- les descendances de 7 mâles ont toutes été thélygènes pures (en tout, $834 q 9$ ), - les 13 autres mâles ont donné 51 descendances se répartissant en 49 amphogènes homogènes (900 $\hat{O} \hat{O}, 974 \hat{q}+$ ) et 2 descendances arrhénogènes exceptionnelles

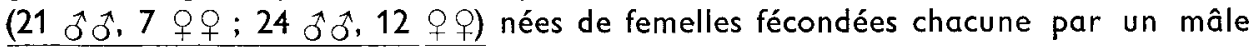
ayant engendré, avec ses autres femelles, des descendances amphogènes.

On retrouve ici, quantitativement, le type de disjonction observé en $4 a$, mais qualitativement très différent : un tiers des mâles induisant la thélygénie ef les $2 / 3$ l'amphogénie.

Les mâles étant les mêmes qu'en $4 a$, il est évident que la différence avec les résultats précédents provient de la femelle qui, rappelons le, est homogamétique ( $2 \mathrm{~A} X X)$. Comme aucun phénomène de monogénie épigénétique n'a été observé à Poitiers, ainsi qu'en témoigne l'amphogénie stable de cette population, on doit orienter l'explication des descendances thélygènes vers un mécanisme génétique (hétérosomique). L'explication la plus simple est que la $\&$ poitevine offre des déterminants sexuels épistałiques vis-à-vis des déterminants de certains mâles existant dans toute descendance arrhénogène. De tels mâles étant incapables d'induire une arrhénogénie génétique dans un croisement consanguin, ils doivent se retrouver, en $4 a$, pour moitié dans le lot des mâles donnant essentiellement de l'amphogénie, et être remplacés, 
en $4 b$, parmi les mâles " amphogènes 》, par les mâles (Ar) des descendances $4 a$. Ce ne sont, dès lors, pas 2 types de mâles qui doivent exister dans une descendance arrhénogène, mais 3 , en nombres sensiblement égaux.

5. Filiation des descendances amphogènes hybrides.

A partir de descendances amphogènes obtenues au $\S$ III, des croisements consanguins - où chaque mâle est croisé avec 2 à 3 femelles sœurs - ont été effectués, que l'on peut classer en 9 ensembles d'après l'origine des génifeurs. Les résultats sont les suivants :

1er ensemble $\left(F_{4} A / T 6 B\right): 4$ descendances $(A r), 14$ amphogènes

$2^{\mathrm{e}} \quad-\quad\left(\mathrm{F}_{4} \mathrm{~A} / \mathrm{T} 1\right): 1(\mathrm{Ar}), 33$ amphogènes

$3^{\mathrm{e}} \quad-\quad\left(F_{4} A / T 2\right): 0(A r), 7$ amphogènes

$4^{\mathrm{e}} \quad-\quad\left(F_{4} A / T 5\right): 0(A r), 18$ amphogènes

5e $\quad-\quad\left(F_{5} A / T 3 B\right): 2$ (Ar), 3 amphogènes

6e $\quad-\quad\left(F_{5} A / T 1 A\right): 0(A r), 5$ amphogènes

$7 \mathrm{e} \quad-\quad\left(F_{5} A / T 6\right): 4(A r), 9$ amphogènes

$8 \mathrm{e} \quad-\quad\left(F_{5} A / T 6^{\prime}\right): 7(A r), 21$ amphogènes

ge $\quad-\quad\left(F_{7} 10\right.$ B 1 C 5 B) : 12 (Ar), 28 amphogènes.

Ces 9 ensembles sont hétérogènes $\left(\chi^{2}=20,05-D L=8\right)$. On doit pouvoir distinguer des descendances marquées par un maintien de l'amphogénie, de celles qui comportent une disjonction entre arrhénogènes et amphogènes, comme pour les résultats de l'inbreeding.

Lorsqu'intervient une disjonction en descendances amphogènes et arrhénogènes (du type $3 \delta^{\star} \delta / 1$ 우 avec parfois un léger excès de femelles), à l'inverse de ce qui a été observé pour les filiations arrhénogènes, les descendances d'un même mâle avec plusieurs femelles peuvent être hétérogènes, groupant à la fois des arrhénogènes et des amphogènes. Ceci prouve que dans ce type de croisement, l'arrhénogénie ne dépend pas que du mâle. Il doit exister, de ce fait au moins 2 types de femelles, et autant de types de mâles puisqu'il y a amphogénie.

On retrouve, parmi les descendances non arrhénogènes, de rares distorsions de la sex-ratio vers une tendance thélygène ou arrhénogène, ainsi qu'une descendance

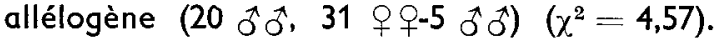

6. Filiations des différents types de thélygénie.

6a. Cas de la thélygénie pure.

Nous avons vu (en III.3a) que le croisement du $\hat{o}$ (Ar) no 3 avec une femelle issue d'une descendance thélygène pure ne modifie pas le caractère arrhénogène de la descendance du mâle, et qu'à la $F_{4}$ (cf. III .4a) intervient une disjonction en descendances arrhénogènes et amphogènes, avec apparition d'une descendance thélygène comportant un mâle exceptionnel.

- Ce mâle exceptionnel a été croisé avec 9 de ses sœurs. Les descendances obtenues constituent un ensemble homogène $\left(\chi^{2}=5,84-\mathrm{DL}=8\right)$ comportant $237 \sigma^{\lambda} \sigma^{*}$ et $670 q q$, ce qui ne diffère pas significativement d'une proportion des sexes de $1 \hat{0} / 3$ 우. La manière la plus simple d'interpréter ces résultats est d'admettre que le 
mâle exceptionnel est issu de l'inversion sexuelle d'une femelle hétérogamétique, 2 A WZ. L'équation des croisements consanguins est :

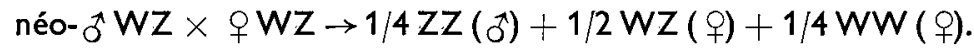

- Cette interprétation a été confirmée en effectuant $20 \mathrm{~F}_{2}$ (10 fois $1 \AA \times 2$ 우 sœurs). Les descendances se sont bien réparties en 14 amphogènes homogènes $\left(\chi^{2}=16,42-D L=43\right)$ ef 5 thélygènes avec, chez l'une, un mâle exceptionnel. Un même géniteur a présenté, dans trois cas, une descendance thélygène avec une femelle, amphogène avec l'autre, montrant que c'est bien la femelle qui est reponsable de la thélygénie. La valeur assez élevée du $\chi^{2}$ des descendances amphogènes provient de l'existence d'une descendance à tendance $(T)\left(32 \hat{o} \hat{O}^{\hat{0}}, 54\right.$ 우 ) et d'une autre à tendance (Ar) (29 $\hat{\sigma} \hat{\sigma}, 12$ 우), cette dernière issue du géniteur de la descendance (T) comportant 1 ô exceptionnel.

- Le mâle père en question a été croisé, l'année suivante, avec 4 des femelles qui avaient offert des descendances (T) pures, en étant croisées avec d'autres mâles. Chez 2 de ces 4 femelles, sont alors apparus des mâles exceptionnels $(3 \hat{o} \widehat{o}, 143$ q $q$; 1 of, 125 웅). Ce test n'est cependant pas significatif.

- Une des génitrices des descendances $(T)$ de la $F_{2}$ a été croisée, l'année suivante, avec un ồ reconnu comme (Ar), car ayant engendré (en $F_{5} 10$ B 1 A) 3 descendances

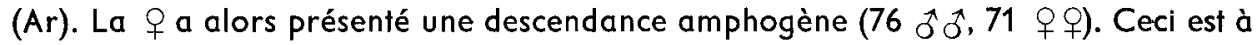
rapprocher des résultats obtenus en croisant un $\hat{\sigma}\left(\mathrm{Ar}\right.$ ) (exemple le $\delta^{\hat{\sigma}} \mathrm{n}^{\circ} 3$, en III. 3a) avec une $q$ poitevine ou $\mathrm{TS}_{5}$.

6b. Filiations des descendances à tendance thélygène.

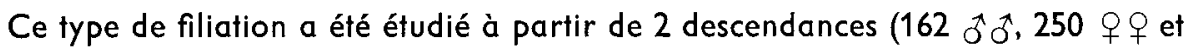
70 ô $\sigma^{\circ}, 140$ 우) de la $F_{5} 10$ B 1 A (cf. III. 4a) :

- en $F_{6} 10$ B 1 A 2 A, 10 mâles ont été croisés chacun avec 2 femelles. La plupart des descendances ( 12 sur 17 fécondes) sont amphogènes; 2 sont allélogènes, 2 à

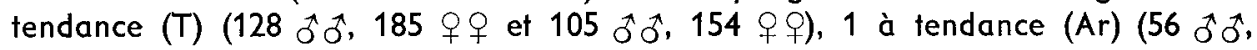
36 우), 1 est (T) pure ; enfin 1 est fortement (Ar) : 70 oิ 0 , 7 , différant significativement du rapport 3 ô $\delta / 1$ 우 ;

- en $F_{6} 10 \mathrm{~B} 1 \mathrm{~A} 2 \mathrm{C}$, le même type de croisement a abouti à 9 amphogènes, 7 à tendance thélygène, qui constituent un ensemble homogène avec 37,1 p. 100 de mâles ; 4 sont arrhénogènes, dont une pure $(37 \hat{o} \hat{\delta})$ et les 3 autres fortes $(20 \hat{d} \hat{d}$.

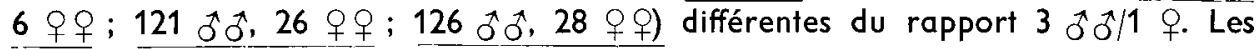
descendances d'un même $\hat{\sigma}$ sont fréquemment hétérogènes : $(A)$ ou $(T)$ et $(A r)$; - en $F_{6} 10 \mathrm{~B} 1 \mathrm{~A} 10 \mathrm{~A}: 5$ descendances sont amphogènes et 5 à tendance thélygène, homogènes, à 38,9 p. 100 de mâles ;

$-20 \mathrm{~F}_{7}$ effectuées à partir d'une $\mathrm{F}_{6}$ précédente à tendance $(T)\left(75 \delta^{\star} 0^{\star}, 108\right.$ q $q$ ) se répartissent en $10(\mathrm{~A})$ et 11 à tendance $(\mathrm{T})$;

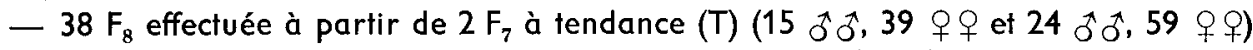
ont donné : $23(\mathrm{~A}), 9$ à tendance $(\mathrm{T}), 2$ allélogènes, $4(\mathrm{Ar}): 74 \hat{\delta} \hat{\delta}, 10$ 우우 ; $20 \hat{\delta} \hat{\delta}$. 2 우우; $17 \hat{o \hat{o}} ; 36 \hat{\jmath} \hat{\jmath}, 7$ 우우.

La descendance de certains mâles est hétérogène, ex $:(T)+(A r)$. Par ailleurs, les 23 descendances (A) constituent un ensemble homogène significativement différent 
de l'amphogénie $\left(\chi^{2}=5,63\right)$, à tendance $(T): 528 \delta \widehat{\partial}, 608$ 우. On peut ainsi considérer que, de la $F_{5}$ à la $F_{8}$, la tendance thélygène a pu être sélectionnée. Un type nouveau de résultat a été par ailleurs obtenu : l'apparition de descendances arrhénogènes pures ou fortes, où les femelles font figure d'exception.

7. Filiation des descendances à arrhénogénie pure ou forte.

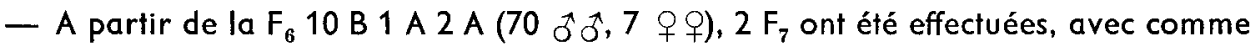

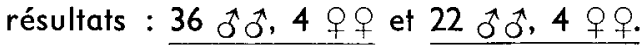

- $7 \mathrm{~F}_{8}$ ont été constituées à partir de la $\mathrm{F}_{7}$. Les résultats sont les suivants : $21 \hat{\delta} \hat{\delta}$;

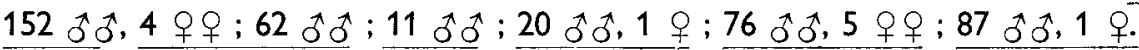

Ainsi, l'arrhénogénie pure ou forte est transmissible. Son origine doit être recherchée dans l'inversion sexuelle de mâles homogamétiques ( $2 \mathrm{~A} Z \mathrm{ZZ}$ ) puisque celle de mâles hétérogamétiques conduirait à une proportion des sexes de $3 \hat{\delta} \widehat{\delta} / 1$ q . Une telle hypothèse va dans le sens de l'équation proposée précédemment pour expliquer l'origine de la thélygénie pure et des mâles exceptionnels $(2 \mathrm{~A} \mathrm{WZ})$.

- Une confirmation de ces hypothèses est donnée par le croisement des mâles ef des femelles exceptionnels apparus dans les 2 types de descendances monogènes. 4 croisements de ce type ont donné, en effet, de l'amphogénie (250 $\hat{\delta} \widehat{\delta}, 231$ q q) qui

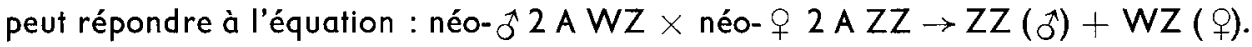
Cette amphogénie s'est maintenue en $F_{2}$.

\section{IV. - Emploi de néo-mâles expérimentaux du type Neuvy dans divers croisements}

1. Origine des néo-mâles.

Des néo-mâles ont été obtenus en implantant des glandes androgènes à de très jeunes femelles de différentes origines, mais toujours issues de la population de Neuvy : - 2 néo-mâles, no 148 ef 157 , ont été créés à partir de la 3 e récolte effectuée à Neuvyen-Dunois ;

- 2 autres néo-mâles $\left(N_{3}\right.$ et $N_{5}$ ) sont issus de la $F_{6} \operatorname{Ar} 7$ amphogène (cf. 1.3c); - 2 autres néo-mâles $\left(N_{17}\right.$ et $\left.N_{18}\right)$ sont issus d'une descendance $T s_{5}$ (amphogène : 21 ैํ, 22 우) prélevée dans le terrarium correspondant (cf. I.4.1).

Ces 6 néo-mâles, de type Neuvy pur, ont acquis leur état fonctionnel environ 1 an après l'implantation, délai comparable à ce qui a été observé à partir de jeunes femelles de la population poitevine de la même espèce (Juchault et Legrand, 1964).

- 9 autres néo-mâles ont été formés à partir de la descendance hybride thélygène pure (39 $q$ ) ) apparue en $\mathrm{F}_{6} \mathrm{Ar} / \mathrm{T} 6 \mathrm{~B}$ (cf. III.3a). Ces néo-mâles, contrairement aux précédents, sont devenus fonctionnels 5 mois après l'implantation de la glande androgène. Une telle précocité est comparable à celle observée dans la population de SaintHonorat de P. d. petiti (Legrand ef al., 1976), à hétérogamétie femelle.

2. Croisements effectués avec ces néo-mâles.

2a. Néo-mâles de Neuvy :

- le néo-mâle no 148 a été croisé avec 6 femelles prélevées dans 2 descendances arrhénogènes $\left(F_{5} A r 4\right.$ et $\left.F_{4} T s_{1}\right)$ (cf. I.4.1). Toutes les descendances ont été thélygènes : 18 우우; 21 우우 ; 91 우우; 12 우우; 1 우; 3 우우; 
- les néo-mâles $N_{3}$ et $N_{5}$, croisés avec des femelles de la descendance amphogène $\mathrm{F}_{5} \mathrm{Ts}_{2} 6 \mathrm{~A}$ ont donné : 25 우; 19 우 ; 36 우;

- les néo-mâles $\mathrm{N}_{17}$ et $\mathrm{N}_{18}$, croisés avec des femelles sœurs, ont donné : 5 우우; 1 우; 15 우우. Le seul résultat immédiatement exploitable est celui qui concerne les néomâles $N_{17}$ et $N_{18}$ : il témoigne de l'homogamétie femelle ( $\left.2 \mathrm{~A} X X\right)$ de la lignée amphogène $T s_{5}$, comparable à celle du $P$. $d$. dilatatus de Poitiers. Les autres résultats témoignent seulement de l'existence d'individus homogamétiques parmi les géniteurs mâles ef (ou) femelles des croisements.

\section{2b. Néo-mâles hybrides Neuvy-Poitiers :}

- 7 des néo-mâles hybrides ont été croisés avec des femelles prises en $F_{6} \operatorname{Ar} / T 4 a$ (amphogène) (cf. III. 3a). Les descendances obtenues, homogènes, renferment au total $154 \delta^{\lambda}, 406$ 우, ce qui ne diffère pas significativement du rapport $1 \delta / 3$ 우우. Ce dernier témoigne à la fois d'une nature hétérogamétique des néo-mâles et des femelles utilisées, l'équation hétérosomique du croisement étant du type proposé en III. 6 .

- Ces mêmes néo-mâles hybrides, croisés avec 7 femelles prises dans une descendance $(\mathrm{Ar})\left(\mathrm{F}_{6} 10 \mathrm{~B}, 1 \mathrm{C}, 5 \mathrm{~B}\right)$ onf donné le même type de résultat : $109 \hat{0} \widehat{\jmath}$, 354 우오.

- II en a été de même avec 7 femelles prises dans une $F_{7}$ à tendance (T) (cf. III. 3b) ; au total : 106 ô어. 303 우오.

- Croisés avec 13 femelles prises dans 2 descendances amphogènes (obtenues en filiation arrhénogène) les néo-mâles ont donné 2 ensembles de descendances : l'un du type 1 t/ 3 o 9 avec 8 femelles, l'autre (T) pur avec 5 femelles.

- Enfin, croisés avec des femelles exceptionnelles apparues en descendance (Ar) (cf. III .7), qui avaient donné au préalable, dans un croisement consanguin, de l'arrhénogénie presque pure, ces néo-mâles ont conduit à de l'amphogénie dans les dernières portées (après disparition des spermatozoïdes du 1er mâle).

Ces résultats tendent à montrer qu'il existe dans les filiations hybrides NeuvyPoitiers étudiées précédemment, soit uniquement des femelles hétérogamétiques, soił à la fois des femelles hétérogamétiques et des femelles homogamétiques. Ces dernières doivent offrir des déterminants sexuels épistatiques sur les déterminants mâles, la thélygénie résultant alors du croisement : $Q W W \times$ néo- $\hat{\sigma}$ $W Z \rightarrow W W($ o $)+W Z($ ( ) $)$, abstraction faite des néo-femelles $2 \mathrm{~A} Z Z$ dont l'existence est confirmée par leur croisement avec les néo-mâles hybrides.

Du fait que la lignée poitevine utilisée pour l'hybridisme offre une homogamétie femelle - que l'on retrouve dans la lignée $T s_{5}$ de Neuvy - elle ne peut être responsable de l'hétérogamétie observée chez les femelles hybrides. II faut bien admettre que la population de Neuvy est elle-même, en réalité, le résultat de l'hybridation de 2 lignées, l'une à homogamétie femelle (et hétérogamétie mâle), l'autre à hétérogamétie femelle (et homogamétie mâle). Cette conclusion rend compte du fait que I'hybridation Neuvy-Poitiers n'a pas modifié les conditions d'apparition et de transmission de l'arrhénogénie et de l'amphogénie observées initialement dans les croisements Neuvy purs. 


\section{V. - Comparaison des valences des déterminants sexuels des populations de Neuvy et de Poitiers}

\section{Croisements entre lignées $T s_{5}$ et poitevine.}

Partant de la conclusion précédente d'une similitude du déterminisme du sexe, par homogamétie femelle (et hétérogamétie mâle) dans la lignée $\mathrm{Ts}_{5}$ de Neuvy et dans celle de Poitiers, il est possible de comparer les valences des déterminants sexuels de ces deux lignées en effectuant leurs croisements dans les 2 sens et en comparant les sex-ratios obtenues *. Il a été effectué 20 croisements dans chaque sens (10 fois $1 \hat{\sigma} \times 2$ q ), ainsi que des croisements d'inbreeding, respectivement en lignée $\mathrm{Ts}_{5}$ ef poitevine.

- Les $20 F_{1}: \hbar T s_{5} \times$ 우 poitevine (lignée marquée par le gène fauve) ont donné un ensemble hétérogène de descendances $\left(\chi^{2}=31,41-D L=19\right)$ comportant $845 \widehat{\jmath} \widehat{\jmath}, 945$ 우우. On y observe - parmi une majorité d'amphogènes - des des-

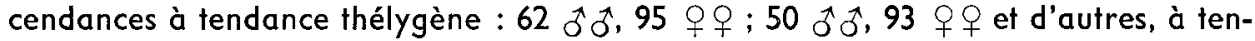
dance arrhénogène : $83 \hat{\sigma} \hat{\sigma}, 61 \%$ 우.

- Les $20 \mathrm{~F}_{1}$ : Õ poitevin $\times$ OTs $s_{5}$ (frères et sœurs des précédents) ont donné un ensemble homogène comportant $754 \hat{o} \hat{\delta} .727$ 우 $\left(\chi^{2}=10,56-\mathrm{DL}=19\right)$ avec une seule

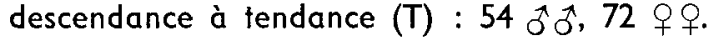

- 20 croisements : $\hat{o} \times T_{T} s_{5}$ onf donné un ensemble homogène comportant 678 oै

- 20 croisements : $\delta \times q$ poitevines (fauves) ont donné un ensemble homogène comportant 733 ô $\delta, 676$ 우.

L'influence de la nature de la femelle est testée en comparant les descendances totales obtenues respectivement avec les femelles $\mathrm{Ts}_{5}: 1432 \hat{\partial} \hat{\sigma}, 1437$ o $ᄋ$ et les femelles poitevines : $1578 \hat{\jmath} \hat{o}, 1621$ 우. Le $\chi^{2}$ est de 0,18 et n'est donc pas significatif : la nature de la femelle n'a pas d'influence sur la sex-ratio de la descendance.

L'influence de la nature du mâle est testée de la même façon, en comparant le total des descendances obtenues respectivement avec les mâles $\mathrm{Ts}_{5}: 1523 \hat{o} \hat{o}$,

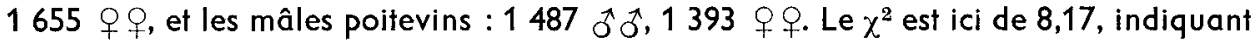
une différence très significative : les mâles $\mathrm{Ts}_{5}$ donnent donc moins de mâles dans leurs descendances que les mâles poitevins, quelle que soit la femelle avec laquelle ils sont croisés.

L'interaction entre lignées est testée par la comparaison entre les totaux des descendances : $\delta \mathrm{Ts}_{5} \times q \mathrm{Ts}_{5}$ et $\partial$ poitevin $\times q$ poitevine, d'une part, $\delta \mathrm{Ts}_{5} \times q$ poi-

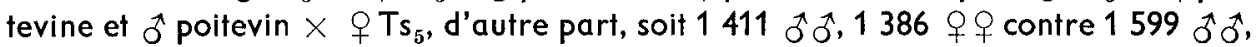
1672 웅. Le $\chi^{2}$ est de 1,41 : il n'y a donc pas d'interaction significative entre les 2 lignées en ce qui concerne la sex-ratio des descendances.

- A partir d'une $\mathrm{F}_{1}\left(\hat{\jmath} \mathrm{Ts}_{5} \times q \mathrm{P}\right)$ à tendance $(\mathrm{T})(62 \hat{o} \delta \hat{\delta}, 95$ 웅), 32 femelles ont été croisées avec des mâles d'une descendance (Ar) pure ( 8 fois $1 \hat{0} \times 4$ 우) : toutes les descendances ont été thélygènes pures. Ceci confirme que toutes les femelles hybrides $\mathrm{Ts}_{5}$-Poitiers ont une constitution $\mathrm{XX}$.

* Dans tous ces croisements et les suivants, les individus $\mathrm{Ts}_{5}$ proviennent de femelles gravides prélevées dans le terrarium correspondant. 
- $20 \mathrm{~F}_{2}$ (10 fois $1 \hat{\delta} \times 2$ q ) ont été effectuées à partir de chacun des 2 types de $F_{1}$, en partant de descendances à tendance thélygène.

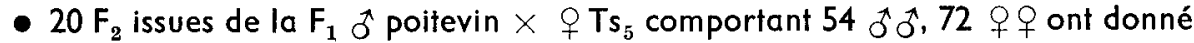
un ensemble homogène de descendances amphogènes : 899 ô 0 , 898 웅. $\chi^{2}=43,76-\mathrm{DL}=38$.

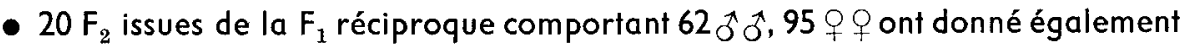
un ensemble homogène de descendances amphogènes : $872 \widehat{\delta} \delta, 860$ 웅. $\chi^{2}=36,81-\mathrm{DL}=42$.

II est à remarquer que dans les 2 types de $F_{2}$, la tendance thélygène a disparu.

S'il existe donc une différence entre les 2 lignées, $T s_{5}$ de Neuvy ef fauve de Poitiers, en ce qui concerne l'action des mâles sur la sex-ratio de leurs descendances, l'amphogénie obtenue en $F_{1}$ dans la grande majorité des descendances et son maintien en $F_{2}$ indiquent que les déterminants sexuels mâles des 2 lignées - $Y_{N}$ et $Y_{P}$ aussi bien que les déterminants femelles $-X_{N}$ et $X_{P}$ - sont de valences comparables.

2. Croisements : ô homogamétique (Neuvy) $\times$ Q poitevine.

L'hétérogamétie femelle mise en évidence en III.6a implique qu'existe à Neuvy une lignée dont les mâles $(2 \mathrm{AZZ}$ ) présentent un déterminant sexuel hypostatique vis-à-vis du déterminant femelle contenu dans l'hétérosome $W$. Ce dernier - d'après ce qui vient d'être dit - peut être aussi bien $X_{P}$ que $X_{N}$ (puisqu'il s'agit d'une filiation hybride) sans que le résultat en soit modifié. On peut vérifier cette épistasie de $X_{P}$ sur le $Z_{N}$ de Neuvy en croisant les mâles des descendances arrhénogènes pures avec des femelles poitevines. On doit retrouver une thélygénie pure, comparable à celle obtenue dans le croisement de femelles poitevines avec $1 / 3$ des mâles issus d'une descendance arrhénogène (cf. III.4b). C'est effectivement ce qu'ont donné 12 croisements ( 6 fois 1 o $\times 2$ 우 poitevines) : 521 우오.

\section{VI. - Reconstitution théorique et expérimentale des filiations $\mathrm{Bi}$ IV observées en inbreeding et outbreeding}

\section{Considérations théoriques.}

1a. Partant des conclusions précédentes concernant les valences sexuelles : $X_{\mathrm{P}}=X_{\mathrm{N}}, \mathrm{Y}_{\mathrm{P}}=\mathrm{Y}_{\mathrm{N}}$ et $\mathrm{X}_{\mathrm{P}}$ ou $\mathrm{X}_{\mathrm{N}}>\mathrm{Z}_{\mathrm{N}}$, on peut reconstituer les équations hétérosomiques des diverses filiations en partant du croisement amphogène correspondant à la fécondation d'une $q$ hétérogamétique par un ổ hétérogamétique : $\left(F_{1}\right)$ o $X Y \times q$

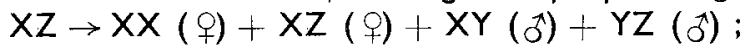

- en $F_{2}$, s'il y a panmixie et si tous les croisements sont viables, on observera une disjonction entre descendances amphogènes et arrhénogènes dans le rapport : $3(A) / 1$ (Ar).
$(A)_{1}:$ o $X Y \times q X X \rightarrow 2 X Y(ð)+2 X X(q)$.
$(A)_{2}: \hat{o} X Y \times$ o $X Z \rightarrow$ idem $F_{1}$.
$(A)_{3}: \delta Y Z \times q X X \rightarrow 2 X Y(\precsim)+2 X Z(q)$.

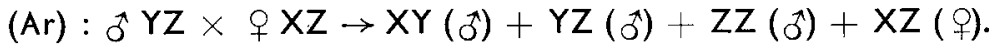


Ainsi, en supposant les 4 types de croisements également fertiles avec chacun 4 individus, sur 16 individus nés en $F_{2}$, se présenteront : 3 우: $X X, 4$ 우: $X Z$,

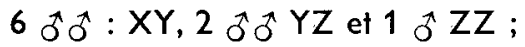

- en $F_{3}$, dans les mêmes conditions de panmixie et de viabilité des croisements, les 3 types de mâles fécondant les 2 types de femelles, on observera la disjonction suivante :

$$
\begin{aligned}
& \Xi X Y \times\left\{\begin{array}{l}
q X X \rightarrow \text { idem }(A)_{1} \text {, probabilité : } 18 / 63 \\
q X Z \rightarrow \text { idem } F_{1}(A) \text {, probabilité : } 24 / 6
\end{array}\right.
\end{aligned}
$$

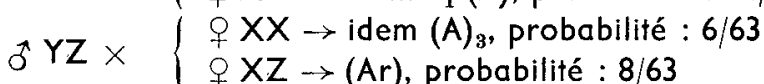

$$
\begin{aligned}
& \sigma Z Z \times\left\{\begin{array}{l}
q X X \rightarrow X Z(q):(T), \text { probabilité }: 3 / 63 \\
q X Z \rightarrow(A)_{4}, \text { probabilité }: 4 / 63
\end{array}\right.
\end{aligned}
$$

soit, sur 63 descendances : $52(\mathrm{~A}), 8(\mathrm{Ar})$ et $3(\mathrm{~T})$.

Les individus constituant ces descendances $F_{3}$ offriront les mêmes génotypes hétérosomiques ( 3 pour les mâles, 2 pour les femelles) qu'en $F_{2}$, et il en sera de même pour toutes les autres filiations qu'il est facile de reconstituer en appliquant les équations précédentes.

1b. En fait, l'analyse génétique de la descendance $B i$ IV a consisté à effectuer des croisements consanguins à l'intérieur d'un type donné de descendance : (Ar) ou (A). On peut en prévoir les résultats d'après le $\S 1 a$.

- Si l'on part, expérimentalement, d'une descendance (Ar), comme il y a 3 types de mâles et 1 seul type de femelle $(X Z)$, quel que soit le numéro d'ordre de la filiation utilisée, on observera en inbreeding, à la génération suivante, une disjonction en : $2(A) / 1(A r)$, les $2(A)$ correspondant à $(A)_{2}$ et $(A)_{4}$.

- si l'on part d'une descendance du type $(A)_{1}$ el que l'on effectue un inbreeding, on obtiendra indéfiniment une lignée $(A)$ du type Poitiers ou $T s_{5}$ c'est-à-dire à homogamétie femelle.

- Si l'on part d'une descendance $(A)_{2}$, on retombera, en inbreeding, à la génération suivante, sur une disjonction $1(\mathrm{Ar}) / 3(\mathrm{~A})$.

- Si l'on part d'une descendance $(A)_{3}$, on retombera, en inbreeding, à la génération suivante, sur une descendance $(A)$ de type $F_{1}$.

- Si l'on part d'une descendance $(A)_{4}$, on obtiendra en inbreeding indéfini, une lignée (A) du type à hétérogamétie femelle.

- Partant de femelles d'une descendance thélygène $(X Z)$, leurs croisements avec les 3 types de mâles donneront :

- avec un $\lesssim X Y \rightarrow$ idem $F_{1}$,

- arec un $\hat{o} \mathrm{YZ} \rightarrow(\mathrm{Ar})$,

- avec un $\hat{o} Z Z \rightarrow \operatorname{idem}(A)_{4}$.

Cet essai de reconstitution des équations hétérosomiques rend compte des principaux résultats obtenus. Effectivement, la disjonction observée à partir d'une descendance (Ar) - aussi bien en inbreeding Neuvy qu'en hybridation Neuvy-Poitiers est de $2(\mathrm{~A}) / 1$ (Ar) (cf. III. 4a).

De même, en filiation amphogène, on observe - en inbreeding comme en outbreeding - soit un maintien de l'amphogénie (ex. $T s_{5}$ ), soit une disjonction 
(Ar)/(A) non significativement différente du rapport 1 (Ar)/3 (A) prévu par la théorie : compris entre 29/75 et 29/87 en outbreeding. Les rares descendances ( $T$ ) observées en filiation (Ar) ne sont cependant pas expliquées par les équations précédentes.

En effet, en l'absence de thélygénie épigénétique, connue chez Armadillidium vulgare mais inconnue chez Porcellio dilatatus, cette thélygénie ne peut être que génétique et provenir du croisement d'un mâle homogamétique $(2 \mathrm{~A} \mathrm{ZZ})$, précisément spécifique des descendances ( $A r)$, et d'une femelle également homogamétique ( $2 \mathrm{AXX}$ ). Or ce type de femelle est absent de la descendance (Ar) qui ne comporte qu'un unique type de femelle, de constitution XZ. Ainsi, ces rares descendances $(T)$ sont dues : ou bien à l'introduction accidentelle d'une femelle apparue dans une descendance (A) du type $F_{1}$ ou $(A)_{1}$, ou bien à une disjonction anormale des hétérochromosomes d'une femelle $X Z$ avec élimination du chromosome $Z$ lors de la méiose, ou bien à l'existence de femelles $X(0)$, dont seuls les gamètes $A X$ sont viables.

2. Evaluation du taux des différents types de mâles présents dans les descendances arrhénogènes ef amphogènes hybrides.

2a. Partant d'une descendance arrhénogène dont les femelles $(q h)$ ont été testées - par croisement avec des néo-mâles $X Z$ - comme étant hétérogamétiques $(X Z)$ (cf. IV. $2 b$ ), ces femelles ont été croisées avec des mâles $T s_{5}$ et poitevins ( 2 fois : $1 \hat{\sigma} \times 2$ 우 $ᄋ$ ). Les descendances, toutes amphogènes et homogènes (141 $\hat{\sigma} \delta$.

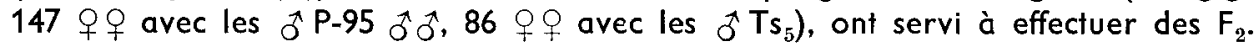

$2 b$. Ces dernières, comportant, à partir de chacun des 2 types de $F_{1}$, respectivement 7 fois et 14 fois $1 \hat{o} \times 2$ 우, ont donné des résultats comparables et conformes aux prévisions du chapitre précédent :

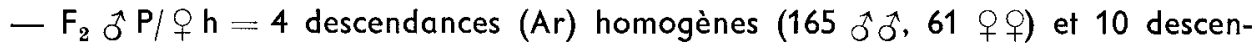
dances (A) (245 $\hat{\sigma} \widehat{O}, 237$ q ) également homogènes.

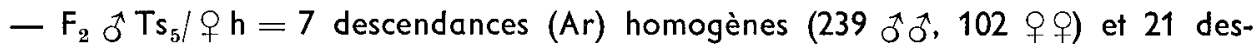
cendances $(A)$ homogènes (728 $\hat{\delta} \hat{\sigma}, 735$ 웅).

Dans les 2 séries, un même mâle a donné, soit 2 descendances de même type : (A) ou (Ar), soit 2 descendances de types différents.

2c. Des $F_{3}$ ont été effectuées à partir de certaines de ces $F_{2}$.

$-10 \mathrm{~F}_{3}\left(5\right.$ fois $1 \mathrm{o}^{\lambda} \times 2$ 우) constituées à partir d'une descendance $(\mathrm{Ar}) \hat{\mathrm{O}^{\lambda}} \mathrm{P} /$ q $\mathrm{h}$ ont donné une disjonction en descendances (Ar) et $(A)$ dans le rapport $4 / 6$.

- Sur $158 F_{3}$ (79 fois $1 \widehat{o} \times 2$ 우) constituées à partir de 13 ensembles de $F_{2}$ amphogènes des 2 séries précédentes :

- dans 8 ensembles, groupant chacun 10 à $20 F_{3}$ (en tout $106 F_{3}$ ), l'amphogénie s'est maintenue ;

- dans les 5 autres ensembles, groupant chacun 10 à $12 F_{3}$, il y a eu disjonction en : 17 descendances ( $\mathrm{Ar}$ ) et 35 descendances (A).

2d. La constitution hétérosomique des mâles présents dans : 3 descendances ( $\mathrm{Ar}$ ) et 2 descendances (A) a été testée en croisant chaque mâle avec 2 femelles : l'une $(q P)$ appartenant à la lignée poitevine, donc homogamétique $(2 \mathrm{AXX})$, l'autre $(q h)$ hétérogamétique $(2 \mathrm{AXZ})$, prélevée soit dans une $F_{3}(A r)$ soit dans une $F_{3}(T)$ 
pure du type $\delta^{\star 2} 2 \mathrm{AZZ} \times \$ 2 \mathrm{~A} \times \mathrm{X}$. Conformément aux prévisions du chapitre $1 b$, les résultats des croisements seront fonction de la constitution du mâle :

- un mâle $X Y$ donnera de l'amphogénie avec les 2 types de femelles,

- un mâle $Y Z$ donnera de l'amphogénie avec la $q P$, de l'arrhénogénie avec la $Q h$,

- un mâle $Z Z$ donnera de la thélygénie avec la $Q P$, de l'amphogénie avec la $Q h$.

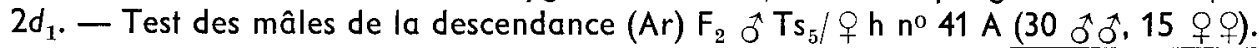

Sur 8 testés : 3 n'ont donné que de l'amphogénie : $X Y ; 4$ ont donné des descendances $(A r)$ et $(A): Y Z ; 1$ a donné : 1 descendance $(A)$ et $1(T): Z Z$.

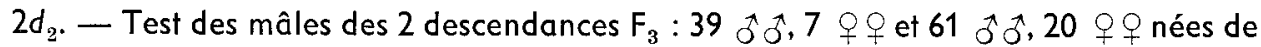

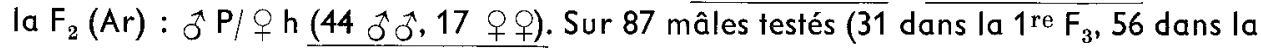
seconde) les résultats, homogènes, ont été additionnés : -27 (respectivement : 8 et 19) n'ont donné que des descendances $(A): X Y ;-28$ (respectivement : 10 et 18) ont donné des descendances (Ar) et $(A)$ : $Y Z ;-32$ (respectivement 13 ef 19) ont donné des descendances $(A)$ et $(T): Z Z$.

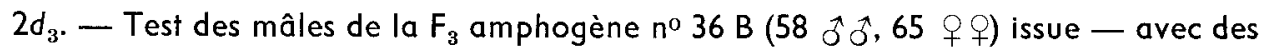

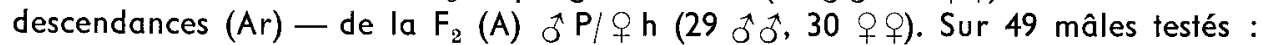
32 n'ont donné que des descendances $(A): X Y$; 16 ont donné des descendances (Ar) et $(A): Y Z$

$2 d_{4^{*}}$ - Test des mâles de la $F_{3}$ amphogène no $40 \mathrm{~B}\left(66 \delta \sigma^{\lambda}, 69\right.$ q ) née de la même $\mathrm{F}_{2}$ que la précédente. Sur 48 mâles testés : 27 n'ont donné que des descendances $(A)$ : $X Y$; 21 ont donné des descendances (Ar) et $(A)$ : $Y Z$.

La $F_{2}$ ayant donné, en $F_{3}$, des descendances $(A)$ et $(A r)$, devait correspondre au type $(A)_{2}$ (ou $F_{1}$ ). En $F_{3}$, doivent apparaître 3 types de descendances $(A)$, dont 2 ne comportent qu'un seul type de mâle $(X Y)$ et l'autre : 2 types de mâles ( $X Y$ ef $Y Z$ ). Les $2 F_{3}$ testées appartiennent à cette dernière catégorie. Néanmoins, dans la $F_{3} n^{0} 36 B$, il y a un déficit significatif des mâles $Y Z\left(\chi^{2}=5,33\right)$ qui ne s'observe pas dans l'autre $F_{3}$ (bien que leur ensemble soit homogène) $\left(\chi^{2}=0,85\right)$. Ce fait peut expliquer la déficience en descendance $(A r)$ parfois observée en $F_{2}$ d'un croisement $(A)_{2}$ ou (Ar).

\section{VII. - Filiation des croisements entre descendances Bi IV de Neuvy et population insulaire de Saint-Honorat de $P$. dilatatus petiti}

\section{Croisements entre lignée $T s_{5}$ de la filiation $B i I V$ et population de Saint-Honorat.}

1a. $F_{1}$ du croisement : $\widehat{\jmath}$ insulaire $\times q T_{s_{5}} . \quad 4$ croisements de ce type - dont les génitrices $\left(\mathrm{F}_{4} 9 \mathrm{~A}\right)$ sont à l'origine de la lignée intitulée $T s_{5}$ - ont donné respectivement : $42 q q$ (en 3 portées), $19 q 9$ en 4 portées, 2 qq (une seule portée), $42 q q$ en 4 portées. La fertilité moyenne est de $9,08 \pm 1,62$. De nombreux embryons avortent au stade de périblastula.

1b. Croisement en retour $\left(\mathrm{CRF}_{2}\right)$ : $q$ hybride $1 \mathrm{a} \times \hat{\jmath}$ insulaire. 3 mâles insulaires ont été croisés, chacun avec 3 à 5 q $q$ hybrides. Les résultats sont les suivants : - avec le $\hat{o} n^{\circ} 1$, une seule $Q$ a donné un descendant $=1 \hat{\jmath} ;-\operatorname{arec}$ le $\hat{\jmath} n^{\circ} 2$, 3 우 n'ont eu aucune descendance, les 2 autres ont donné chacune un seul

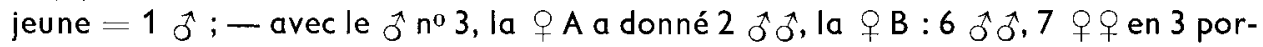

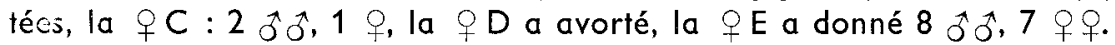


1c. Croisement en retour : q hybride $1 \mathrm{a} \times \widehat{\widehat{T}} \mathrm{Ts}_{5} .10$ croisement $(2$ fois : $1 \hat{\sigma} \times 5$ q ) utilisant des mâles frères des génitrices de la $F_{1}$ ont donné des descendances amphogènes homogènes, dont la fertilité est normale, en tout : 677 ot. 646 우우.

1d. $\mathrm{F}_{1}$ du croisement : $\delta \mathrm{Ts}_{5} \times q$ insulaire.

$1 d_{1}$. - 10 croisements de ce type ont été réalisés en utilisant des mâles pris en $F_{5}$ $T_{5} n^{\circ} 6$ (6 mâles) et no 8 (4 mâles). Dans les 2 séries, il a été observé une disjonction en descendances $(\mathrm{Ar})$ et $(\mathrm{A})$. En tout : 7 (Ar) homogènes $(190 \hat{\sigma} \delta, 74 q 9)$ et $4(\mathrm{~A})$

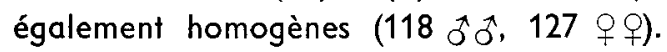

$1 d_{2}$. -12 croisements analogues ont utilisé des mâles prélevés soit en $F_{4} T s_{5}$ ( 3 mâles), soit en $F_{5} T_{5}$ (1 mâle), soit en $F_{6} T s_{5}$ (8 mâles). A part deux croisements du 1 er type, dont un n'a donné que 2 $q$ 우 ef l'autre a avorté, tous les autres ont conduit à des descendances amphogènes. Néanmoins, avec les mâles $F_{6} T s_{5}$, l'ensemble homogène des 8 descendances $\left(\chi^{2}=4,79-\mathrm{DL}=7\right)$ offre une tendance thélygène : $154 \hat{\delta} \widehat{o}$, 225 우우.

1e. Croisement en retour : $q$ hybride $1 \mathrm{~d} \times \widehat{\sigma} \mathrm{Ts}_{5}$.

$1 e_{1}$. -18 croisements de ce type réalisés en utilisant des mâles de $l a F_{6} T s_{5}$ et les femelles prélevées dans une $F_{1} d_{2}$ amphogène ont donné des descendances ampho-

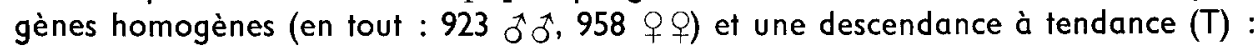
42 ๙ึ。, 70 웅.

$1 e_{2}$. -12 croisements analogues ont utilisé des mâles de même origine et des femelles prises dans une $F_{1} d_{1}$ arrhénogène ; 11 des descendances ont été amphogènes et homogènes (en tout : $647 \hat{\partial} \hat{\sigma}, 694$ 우 ㅇ) ; la dernière offre une tendance (Ar) : $138 \hat{\delta} \hat{o}$, 99 우.

1f. Croisement en retour : $q$ hybride $1 d \times \delta$ insulaire. 15 croisements de ce type ont été réalisés.

$1 f_{1}$. - Les 2 premiers, utilisant les 2 femelles qui constituent l'unique descendance

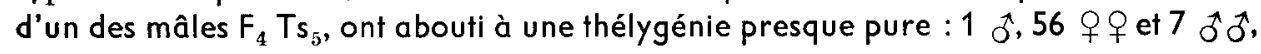
255 우우.

$1 f_{2^{\circ}}-13$ croisements du même type ont utilisé des femelles prélevées :

— soit dans une $F_{1} d_{2}$ amphogène $(1 \hat{\delta} \times 3 q$ 우), avec des résultats comparables aux

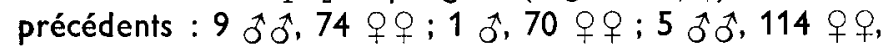

- soit dans les $F_{1} d_{2}$ à tendance $(T)$ ( 5 fois $1 \hat{\sigma} \times 2$ Q $Q$ ) ; un des mâles a donné :

42 우 et $3 \widehat{\delta} \widehat{\delta}, 252$ 우, les autres ont conduit à la disjonction : 1 descendance $(A)$,

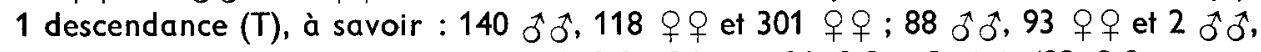

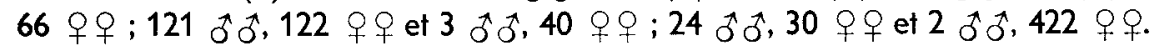

1g. Croisement en retour : $\widehat{o}$ hybride $1 d \times$ 우 insulaire. Les mâles ont été prélevés en $F_{1} d$ arrhénogène $\left(33 \delta \delta^{\star}, 14 q q\right) .12$ croisements $(6$ fois $1 \hat{\delta} \times 2$ q $q)$ n'ont donné que 9 descendances ( $1 \hat{\sigma}$ ef 1 q se sont montrés stériles) ; 3 mâles n'ont offert que des descendances (Ar), les 2 autres, des descendances (A). Les descendances (Ar) sont homogènes et ne diffèrent pas significativement du rapport $3 \delta \delta / 1$ \& (en tout :

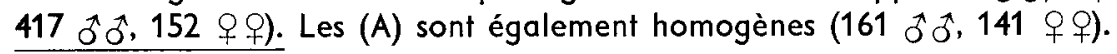


2. Croisements entre hybrides Neuvy-Poitiers et population de Saint-Honorat.

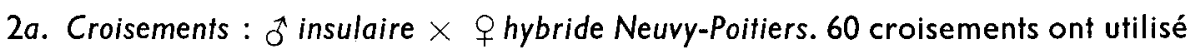
des femelles prélevées dans diverses descendances étudiées au $\S \mathrm{lll}$.

- 10 femelles, issues d'une $F_{4} A r / T$ arrhénogène ont été croisées avec des mâles insulaires. Seules, 4 d'entre elles ont eu une descendance : 3 n'ont eu chacune que

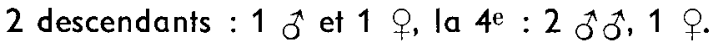

- 20 autres femelles de filiation $(\mathrm{Ar}) \mathrm{F}_{6} 10 \mathrm{~B} 1 \mathrm{C}$ - dont les sœurs ont donné, avec les néo-mâles physiologiques hybrides, des descendances du type $1 \delta / 3 q q$ - ont presque toutes avorté, 2 d'entre elles n'ayant qu'un seul descendant, l'une : 1 శ, l'autre : 1 q.

- 10 femelles, nées en $F_{4} A r / T$ thélygène et 20 autres, issues d'une $F_{7}$ à tendance (T) ont avorté.

2b. Croisements : $\delta$ hybride Neuvy-Poitiers $x q$ insulaire.

$2 b_{1}$. - Des géniteurs de certaines descendances (Ar) : $F_{7} 10$ B 1 C 5 B, croisés chacun avec 2 femelles insulaires onf donné : l'un, 2 descendances (Ar) (au total, 64 ô ô.

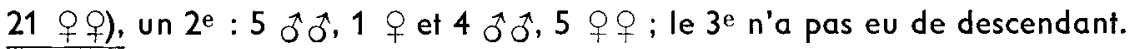

$2 b_{2}$. - Des mâles prélevés dans une descendance (Ar) $\left(F_{7}\right.$ Neuvy-Poitiers) issue du $2 \mathrm{e}$ mâle $2 b_{1}$ ont donné respectivement avec des femelles insulaires : $7 \hat{\sigma} \hat{o}, 1+q$;

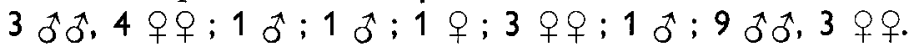

$2 b_{3}$. - Des géniteurs de la $F_{6}$ à tendance $(T) 10 \mathrm{~B} 1 \mathrm{~A} 10 \mathrm{~A}$ ont été croisés chacun avec 2 femelles insulaires; les descendances obtenues sont amphogènes et homogènes (en tout : $208 \delta \widehat{\partial}, 194$ 웅).

$2 b_{4}$. - Les fils des mâles précédents ( $F_{7}$ Neuvy-Poitiers), croisés avec des femelles insulaires ont également donné des descendances amphogènes homogènes (en tout, 262 ฮึ đ. 267 웅).

\section{Discussion.}

Les résultats obtenus, qui peuvent être comparés à ceux des croisements entre la population poitevine de $P$. $d$. dilatatus et celle de Saint-Honorat de $P$. d. petiti (Legrand et Juchault, 1974), doivent être examinés sous deux aspects : d'une part, pour les renseignements qu'ils fournissent sur la constitution hétérosomique des individus de la filiation Neuvy, d'autre part, en ce qui concerne la fertilité variable des croisements.

3a. Données apportées sur la constitution hétérosomique des individus de la filiation $\mathrm{Bi}$ IV.

$3 a_{1}$. Constitution de la lignée $\mathrm{Ts}_{5}$.

- Si l'on fait provisoirement abstraction de leur faible fertilité, les $F_{1} 1 a$ à thélygénie pure, correspondent au croisement d'un mâle connu pour être homogamétique ( $2 \mathrm{~A} Z_{i} Z_{i}$ ) avec une femelle également homogamétique, dont les déterminants sexuels sont épistatiques sur ceux du mâle : ô $Z_{i} Z_{i} \times q X_{N} X_{N} \rightarrow X_{N} Z_{i}(q)$. Ainsi, l'hétérosome $Z_{i}$ du mâle insulaire est-il comparable au $Z_{N}$ de certaines lignées de la population de Neury. 
- Les $C_{2} F_{2} 1 b$, amphogènes, confirment cette conclusion, ils peuvent être représentés par l'équation : $q X_{N} Z_{i} \times \delta Z_{i} Z_{i} \rightarrow X_{N} Z_{i}(q)+Z_{i} Z_{i}(\delta)$. Si les résultats de ces 2 types de croisements confirment donc l'état homogamétique de certaines femelles de la lignée $\mathrm{Ts}_{5}$ de Neuvy, $\mathrm{F}_{4} 9 \mathrm{~A}$, ceux qui découlent de l'utilisation de mâles $\mathrm{Ts}_{5}$ sont hétérogènes.

- Les $F_{1} 1 d_{1}$, offrant une disjonction (A)-(Ar) ne peuvent s'interpréter, en fonction de l'analyse du $\S \mathrm{VI}$, qu'en admettant l'existence de 2 types de mâles dans certaines filiations $T s_{5}$, à savoir : $F_{5} T s_{5} n^{\text {os }} 6$ et 8 :

- des mâles $Y_{N} Z_{N}$, responsables des descendances (Ar) :

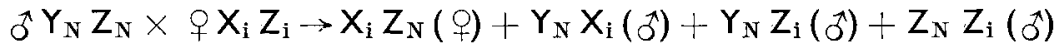

- des mâles $X_{N} Y_{N}$, responsables des descendances $(A)$ :

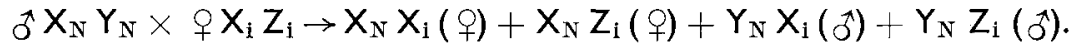

- En $F_{1} 1 d_{2}$, l'amphogénie de toutes les descendances est imputable à l'existence d'un seul type de mâle $\left(X_{N} Y_{N}\right)$. Quant à l'apparition de 2 types de femelles hybrides dans ce croisement, elle est démontrée par les $\mathrm{CRF}_{2}$ if :

- les descendances thélygènes proviennent des femelles homogamétiques : $q \mathrm{X}_{\mathrm{N}} \mathrm{X}_{\mathbf{i}} \times \delta \mathbf{Z}_{\mathbf{i}} \mathbf{Z}_{\mathbf{i}} \rightarrow \mathrm{X}_{\mathrm{N}} \mathbf{Z}_{\mathbf{i}}+\mathrm{X}_{\mathrm{i}} \mathbf{Z}_{\mathbf{i}}\left(\begin{array}{l}+ \\ +\end{array}\right)$

- les descendances amphogènes proviennent des femelles hétérogamétiques : o $\mathrm{X}_{\mathrm{N}} \mathbf{Z}_{\mathrm{i}} \times \delta \mathrm{Z}_{\mathrm{i}} \mathrm{Z}_{\mathrm{i}} \rightarrow \mathrm{X}_{\mathrm{N}} \mathrm{Z}_{\mathrm{i}}($ o $)+\mathrm{Z}_{\mathrm{i}} \mathrm{Z}_{\mathrm{i}}(\delta)$.

II faut noter l'inversion sexuelle spontanée en néo-mâles de certaines femelles $X_{N} Z_{i}$ ou $X_{i} Z_{i}$, visible dans les descendances thélygènes, mais intervenant peut être dans des descendances amphogènes.

- Les mâles $T s_{5}$ utilisés dans les autres croisements :

$\mathrm{CRF}_{2} 1 \mathrm{c}$ et $1 \mathrm{e}$, provenant de la $\mathrm{F}_{6} \mathrm{Ts}_{5}$, doivent appartenir également à 1 seul type $\left(X_{N} Y_{N}\right)$, qui rend compte de l'amphogénie observée.

Ainsi, les croisements réalisés entre la filiation $\mathrm{Ts}_{5}$ de Neuvy et la population insulaire viennent compléter les données acquises dans les paragraphes précédents, montrant qu'à l'origine, la filiation $\mathrm{Ts}_{5}$ correspondait à une hybridation entre 2 lignées : l'une à homogamétie femelle, l'autre à hétérogamétie femelle. En se rapportant aux équations théoriques exposées au $\S \mathrm{VI}$, on peut reconstituer cette origine de la façon suivante :

La $F_{1} B i$ IV pouvait correspondre au croisement : of $X_{N} Y_{N} \times q X_{N} Z_{N}$; la $F_{2} n^{\circ} 9$ : au type $(A)_{2}$, identique à la $F_{1}$, la $F_{3} n^{\circ} 9$ de même ; en $F_{4} n^{\circ} 9$, le nombre suffisant de descendances a permis la réalisation de la disjonction (Ar)-(A), et la descendance intitulée $T s_{5}$ devait également être du type $(A)_{2}$ : c'est ce qui éclaire un résultat resté jusqu'ici sans explication : la disjonction en descendances (A) et (Ar) observée en III. $2 a$ avec le $\hat{o}(A r) n^{\circ} 4$, les descendances (Ar) devant correspondre à 2 femelles $F_{5} T s_{5}$ hétérogamétiques $\left(X_{N} Z_{N}\right)$, alors que toutes les autres $\left(3\right.$ avec le ô $n^{\circ} 3$, 1 avec le ô $n^{\circ} 4$ ) étaient homogamétiques $\left(X_{N} X_{N}\right)$. Les $F_{6}$ et $F_{7}$ réalisées, ainsi que les croisements intervenus dans le terrarium, ont dû correspondre à des descendances essentiellement du type $(A)_{1}$, ce qui explique l'homogamétie femelle $X_{N} X_{N}$ et l'hétérogamétie mâle $X_{N} Y_{N}$ observées dans tous les croisements ultérieurs utilisant la lignée $T s_{5}$. 
$3 a_{2}$. - Constitution des hybrides Neuvy-Poitiers.

- Les $F_{1} 2 a$ entre mâles insulaires et femelles hybrides Neuvy-Poitiers, si l'on fait abstraction de leur très faible fertilité, ont apporté un résultat inconnu lors des croisements réalisés entre populations poitevine et insulaire, à savoir une amphogénie

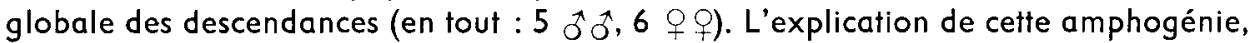
confirmant les conclusions de l'emploi de néo-mâles physiologiques au $\S \mathrm{IV} .2 b$, réside en l'état hétérogamétique $(X Z)$ des femelles hybrides :

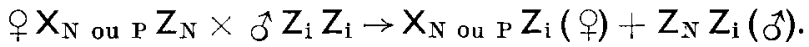

- Les $F_{1}$ entre femelles insulaires et mâles hybrides Neuvy-Poitiers confirment l'existence de mâles (Ar) : YZ en $2 b_{1}$. Par contre, l'infertilité relative des $F_{1} 2 b_{2}$ ne permet pas de retrouver d'une façon nette l'existence de différents types de mâles $(X Y, Y Z, Z Z)$ en descendance (Ar).

- Les $F_{1} 2 b_{3}$ et $2 b_{4}$ confirment l'absence de mâles $Y Z$ dans les descendances à tendance thélygène et apportent un résultat nouveau : la disparition totale de la tendance (T) dans la descendance issue de femelles insulaires montre que la femelle hybride Neuvy-Poitiers est en grande partie responsable de cette forme de thélygénie.

3b. Mécanisme de l'infertilité de certains croisements. Nous avons pu (Legrand ef al., 1979, sous presse) rapporter l'infertilité des croisements entre mâle insulaire ef femelles poitevines ou femelles hybrides Poitiers-Saint-Honorat d'ascendance maternelle poitevine, à l'absence d'un bactéroïde symbiote dans la population de Poitiers de $P$. d. dilatatus, alors qu'il est présent dans les ovocytes et les cellules folliculaires des $P$. $d$. petiti insulaires.

- La plupart des croisements infertiles observés dans la présente étude répondent aux mêmes types que ceux de la publication précédente : $-F_{1} 1 a$ : $\hat{0}$ insulaire $x q$ $\mathrm{Ts}_{5} ;-\mathrm{CRF}_{2} 1 b: \hat{o}$ insulaire $\times \phi$ hybride de mère $\mathrm{Ts}_{5} ;-\mathrm{F}_{1} 2 a: \hat{o}$ insulaire $\times q$ hybride Neuvy-Poitiers. L'état homo- ou hétérogamétique de la femelle n'a pas d'influence sur l'infertilité. Cette dernière est amplifiée, de la $F_{1} 1 a$ au $C R F_{2} 1 b$ par un renforcement du génotype petiti dans les zygotes issus de ce $\mathrm{CRF}_{2}$. L'infertilité est également très forte lorsque la femelle est hybride Neuvy-Poitiers ( $\left.F_{1} 2 a\right)$.

- Au contraire, on observe généralement une fertilité normale lorsqu'un mâle de Neuvy - ou hybride Neuvy-Poifiers - est croisé avec une femelle insulaire, ou une femelle hybride Neuvy-Saint-Honorat d'ascendance maternelle insulaire $\left(\mathrm{CRF}_{2} \mathrm{e}_{\mathrm{e}}\right.$ et $1 \mathrm{~g}$ ). De même, la fertilité est normale lorsque le mâle insulaire est croisé avec une + hybride d'ascendance maternelle insulaire $\left(\mathrm{CRF}_{2} 1 f\right)$, que l'étude précédente (1979) a montrée pourvue du symbionte, transmis par le cytoplasme de l'ovocyle, mais semblant disparaître chez les mâles.

Or, l'examen ultrastructural des ovaires des femelles de Neuvy homo- et hétérogamétiques n'a révélé aucun bactéroïde symbiote. On peut donc invoquer ici le même mécanisme de l'avortement des embryons - quel que soit leur sexe - au stade périblastula : une incompatibilité entre génotype petiti et cytoplasme dépourvu du symbionte, susceptible d'inhiber la duplication des chromosomes petiti (qui sortent du fuseau mitotique de segmentation à l'anaphase) et, plus précisément, de certains locus. Les quelques embryons poursuivant leur développement ont été rapportés, dans la publication précédente, à des locus indifférents à la présence d'une substance apportée par le symbionte. 
La fertilité générale des croisements Neuvy-Poitiers et de celle des croisements : § Neuvy ou hybride Neuvy-Poitiers $\times q$ petiti montre que les génotypes des 2 populations continentales s'accommodent aussi bien de la présence du symbionte que de son absence.

Néanmoins, l'infertilité semble parfois l'apanage de certains mâles $T s_{5}$ : en $1 d_{2}$ ( 2 sur 3 issus de $F_{4} T s_{5}$ ), ou hybrides Neuvy-Poitiers (en $2 b_{1}$ ). Dans ce $2^{\mathrm{e}}$ cas, il s'agif d'un caractère transmissible puisque les fils - en lignée Neuvy/Poitiers - offrent le même caractère ; par ailleurs, il y a incompatibilité entre génotype $T s_{5}$ et ovocytes petiti car ces mâles offraient une fertilité normale avec des femelles sœurs. Des cas analogues ont été observés dans les croisements $\widehat{o}$ Poitiers $\times q$ insulaire.

\section{VIII. - Données concernant d'autres individus de la population de Neuvy}

Cette étude ne prétend pas être une analyse exhaustive de la population de Neuvy : il eût fallu pour cela tester systématiquement la constitution hétérosomique d'un grand nombre d'individus en employant certains types de croisements utilisés pour l'éłude de la filiation $\mathrm{Bi}$ IV. Les descendances d'autres femelles de la population de Neuvy ont été essentiellement analysées - à un stade de l'éfude où la constitution hétérosomique de la $q \mathrm{Bi} I V$ n'était pas élucidée - pour voir comment se transmettait le pseudo-hermaphrodisme masculin externe de type $A$, aussi bien en inbreeding qu'en outbreeding. Les résultats obtenus apportent quelques données sur la constitution hétérosomique de 5 autres femelles récoltées dans la station.

1. Descendance de la femelle Bi l. - Quatre générations ont été étudiées en inbreeding.

- A partir de 24 femelles de la $F_{1}$ prises dans les 3 portées, $17 F_{2}$ ont été obtenues (les 7 autres femelles ont avorté) ; le total des descendances, homogène $\left(\chi^{2}=10,64-D L=16\right)$ comporte $170 \delta \delta$. 129 of $q$ et offre une tendance (Ar) significative ; 10 des génitrices de ces $F_{2}$ étaient intersexuées (type $A$ ) ; seules 2 femelles filles ont acquis ce phénotype.

- $31 \quad F_{3}$ ont été constituées, qui ont abouti à un ensemble hétérogène $\left(\chi^{2}=55,4-\mathrm{DL}=30\right)(707 \delta \AA, 791$ 우 $)$ rassemblant une majorité de descendances amphogènes ef quelques-unes à tendance thélygène (ex. $63 \hat{\sigma} \widehat{\sigma}, 96$ q $q ; 18 \hat{\sigma} \delta$. 33 우우) ; 3 femelles filles engendrées par des génitrices non intersexuées, sont devenues intersexuées du type $A$ et 127 ont acquis le phénotype intersexué $B$; ces dernières proviennent indistinctement de génitrices normales ou intersexuées de type $A$. - $27 F_{4}$, effectuées à partir de $F_{3}$ amphogènes ou à tendance $(T)$, ont donné, dans tous les cas, des descendances amphogènes homogènes, au total : $645 \hat{\partial} \partial \hat{\sigma}$, 641 우 ; aucune femelle intersexuée de type $A$ n'est apparue ; il a été dénombré 19 intersexuées de type $B$.

2. Descendance de la femelle Bi VI. - Les individus des deux sexes, nés de la $q$ $B i$ VI, ont été croisés avec des individus de la population poitevine de $P$. $d$. dilatatus.

2. Dans le sens : $Q \mathrm{~F}_{1} \mathrm{Bi} \mathrm{VI} \times ð$ poitevin, ce sont des mâles de phénotype pseudoalbinos (Legrand, 1962) qui ont été croisés avec les femelles fortement intersexuées de la $\mathrm{F}_{1} \mathrm{Bi}$ VI. 22 croisements (10 fois $1 \lesssim \times 2 q$ ) ont tous avorté. 
2. Dans le sens inverse : $q$ poitevine $\times F_{1} B i$ VI, ce sont des femelles poitevines de phénotype fauve qui ont été croisées avec les mâles $\mathrm{Bi}$ VI.

- Sur 13 croisements $F_{1}, 3$ ont totalement avorté, les autres ont offert des descendances très faibles : en tout, $26 \hat{\jmath} \delta, 27$ 웅 $\left(\chi^{2}=7,27-\mathrm{DL}=9\right)$. Une seule des femelles filles (no 4 B) a acquis le phénotype intersexué $A$.

- $9 \mathrm{CRF}_{2}$ (filles $\times \widehat{O}$ père) ont été effectués, ainsi que $7 \mathrm{~F}_{2}$. Les descendances sont également faibles. Si l'on ne retient que celles dont le nombre d'individus est $>20$, on y remarque, en $\mathrm{CRF}_{2}: 2$ amphogènes $(9 \hat{\sigma} \hat{\sigma}, 11$ q $q$ ef $12 \hat{\sigma} \hat{\sigma}, 10 q q$ ) ef une

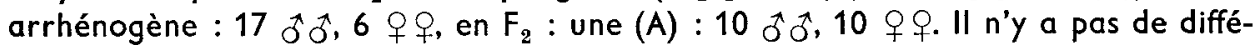
rence significative entre les 2 ensembles $\left(\chi^{2}=21,10-D L=15\right)$, qui ne diffèrent pas de l'amphogénie : $59 \hat{\jmath} \hat{\sigma}, 42$ qq en $\mathrm{CRF}_{2}\left(\chi^{2}=8,72-\mathrm{DL}=8\right)$ contre $38 \hat{\jmath} \hat{\jmath}$. 45 우우 en $\mathrm{F}_{2}\left(\chi^{2}=9,73-\mathrm{DL}=6\right)$.

3. Descendance de la $Q_{\mathrm{Bi}} \mathrm{VIII}$. - Le protocole des croisements a été le même que pour la $q$ Bi VI. Sur 8 croisements dans le sens : ô poitevin (à phénotype fauve) $\times q$ $\mathrm{F}_{1} \mathrm{Bi}$ VIII, 4 seulement ont eu une descendance, d'ailleurs très faible, en tout : 11 ô. 8 우우.

- $6 \mathrm{~F}_{2}$ ont été effectuées ; leur ensemble, homogène, est amphogène : $112 \widehat{\delta} \widehat{\delta}$, 118 우 $\left(\chi^{2}=2,7-\mathrm{DL}=5\right)$.

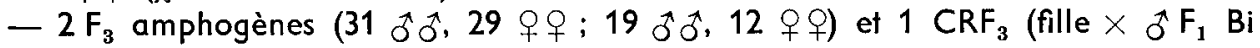
VIII) à tendance (T) : $14 \hat{\delta} \bar{\delta}, 26$ 우, ont été obtenues.

- $3 F_{4}$, effectuées à partir des $F_{3}$ ont été également amphogènes et homogènes : en tout, $40 \widehat{\partial} \delta, 44$ 우; $11 \mathrm{~F}_{4}$ effectuées à partir du $\mathrm{CRF}_{3}$ ont donné un ensemble homogène $\left(\chi^{2}=10,73-D L=10\right)$ comportant $126 \hat{\sigma} \hat{\sigma}, 107 q q$, où l'on observe 2 descendances à tendance (Ar) : $21 \delta \delta, 11$ q $q$ et $20 \delta \tau, 10 \%$, homogènes et ne différant pas significativement du rapport $3 \hat{\delta} \delta / 1+\left(\chi^{2}=2,60\right)$.

4. Descendance de la + $\mathrm{Bi}$ V.

- 6 croisements : $\delta$ poitevin $\times q F_{1} B i V$ ont donné un ensemble amphogène, homogène, en tout : $30 \hat{\sigma} \hat{\sigma}, 26$ q .

- $12 F_{2}$ ont été effectuées, groupées en 5 ensembles d'après l'origine des géniteurs.

- 4 de ces ensembles sont amphogènes et homogènes : en tout $219 \hat{\sigma} \delta .228$ q $q$ $\left(\chi^{2}=10,06-\mathrm{DL}=8\right)$;

- le $5^{\mathrm{e}}$ ensemble, dont les 3 couples de géniteurs descendent du même mâle

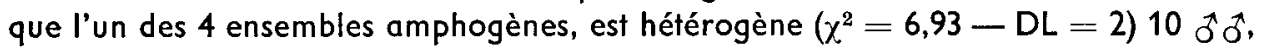

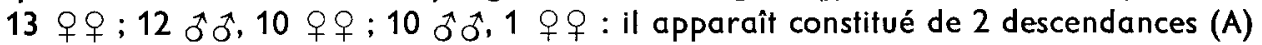
ef d'une $3^{\mathrm{e}}$ qui - bien que faible - est probablement (Ar).

5. Il faut rappeler ici qu'1 néo-mâle physiologique ( $\left.n^{0} 148\right)$ constitué à partir d'une jeune femelle récoltée à Neuvy-en-Dunois au cours du $3^{\text {e }}$ prélèvement, et croisé avec des femelles nées dans 2 descendances (Ar), n'a donné que des femelles (cf. IV.2a).

\section{Discussion.}

a) Le résultat le plus net concerne le néo-mâle physiologique no 148 : la thélygénie pure obtenue à la suite de son croisement avec 6 femelles prélevées en descendance (Ar) ef dont la constitution hétérosomique est donc $2 \mathrm{~A} X Z$, témoigne de la nature homogamétique $(2 A X X)$ de la jeune femelle inversée sexuellement. 
b) En ce qui concerne la femelle $B i$ l, l'absence totale, en 4 générations, de descendances arrhénogènes du type $3 \widehat{\delta} \delta / 1$ ㅇ témoigne de l'absence de mâle $Y Z$, donc d'une hybridation entre lignées respectivement à hétérogamétie mâle et hétérogamétie femelle, et plaide pour une constitution : soit $\delta^{t} \mathrm{XY} \times q \mathrm{XX}$, soit $\delta^{t} \mathrm{ZZ} \times$ 우 $Z X$ du croisement (de la nature) qui a donné naissance à la $F_{1}$.

c) En ce qui concerne la femelle Bi VI, dont la descendance (de la nature) était à tendance (Ar) : 41 ô $\hat{\sigma}, 23$ 우이 le croisement de ses fils avec des femelles poitevines $(X X)$ élimine, par l'amphogénie des résultats, une constitution $Z Z$ des mâles; ces derniers peuvent être, de ce fait, soit $X Y$, soit $Y Z$. Dans le $1^{\text {er }}$ cas, les $F_{2}$ et les $C R F_{2}$ devraient être tous amphogènes; dans le $2^{\mathrm{e}}$ cas ( $\hat{\sigma}^{\mathrm{Y}} Y Z$ ), les $F_{2}$ tous arrhénogènes. Statistiquement, c'est la première hypothèse qui semble confirmée, mais la faible fertilité des descendances laisse planer un doute.

d) En ce qui concerne la femelle BI VIII, l'amphogénie observée dans les $6 F_{2}$ du croisement ô $\mathrm{P} \times$ + $\mathrm{Bi}$ n'élimine pas la possibilité de formation de mâles $Y Z$, le nombre de $F_{2}$ étant trop faible pour considérer comme nulle la probabilité d'apparition d'une descendance (Ar). L'existence de $2 \mathrm{~F}_{4}(\mathrm{Ar}) / 11$ issues du $\mathrm{CRF}_{3}$ peut, au contraire, être une preuve de l'existence de tels mâles. Leur chromosome $Y$ provenant du mâle poitevin, le $Z$ est d'origine maternelle, et la femelle Bi VIll devait donc être hétérogamétique, $X Z$.

e) En ce qui concerne la femelle $B i V$, l'apparition d'une descendance (Ar) en $F_{2}$ du croisement ôt $\mathbf{P} \times$ Q $\mathrm{Bi}$ peut également être rapportée à un mâle $Y Z$ apparu en $F_{1}$, donc à un état hétérogamétique de la femelle $B i \mathrm{~V}$.

Ainsi, malgré le nombre restreint de femelles de la nature analysées, et leur faible fertilité - qui paraît être en partie liée à l'état intersexué de type A puisque la fertilité s'est largement accrue après la disparition de l'intersexualité - les quelques données obtenues vont dans le sens d'une coexistence de femelles homo- et hétérogamétiques dans la population de Neuvy-en-Dunois. Les croisements effectués confirment également le caractère transmissible de cette intersexualité et sa très grande labilité dans les conditions d'élevage au laboratoire. L'apparition en grand nombre de femelles intersexuées du type $B$ dans les descendances nées de l'inbreeding pose le problème d'une corrélation possible entre les deux phénotypes intersexués : le type $B$ pourrait être conditionné par une transformation du facteur infectieux responsable du pseudohermaphrodisme masculin externe de type A, sous l'action de facteurs externes agissant en élevage ef dans les conditions naturelles de vie de certaines populations.

\section{IX. - Conclusion générale}

La présente étude montre qu'à l'intérieur de l'espèce Porcellio dilatatus Brandt, le déterminisme du sexe met en jeu plusieurs types de facteurs.

- Les uns sont très probablement des déterminants sexuels majeurs répartis sur les hétérochromosomes; ces derniers sont indiscernables cytologiquement et encore largement homologues, ainsi que l'ont révélé d'autres études (Legrand ef Juchault, 1974 ; Legrand et al., 1974 ; Artault, 1977).

- D'autres facteurs, susceptibles d'être épigénétiques, parviennent à modifier 
l'expression des gènes sexuels, et provoquent des inversions totales — ou partielles du phénotype sexuel.

1. Variations intraspécifiques du mécanisme hétérochromosomique de détermination du sexe.

L'argument - déjà souligné dans une précédente étude (Legrand et Juchault, 1974) - qui permet de rapporter la détermination sexuelle à un couple d'hétérochromosomes est que les équations classiques fondées sur l'homogamétie d'un sexe et l'hétérogamétie de l'autre sexe rendent parfaitement compte, ici comme précédemment, de la sex-ratio des descendances (amphogène, arrhénogène ou thélygène selon les cas) de tous les croisements effectués - qu'ils mettent en jeu des néo-mâles physiologiques ou des individus de différentes populations. Le présent travail confirme l'existence, chez des populations différentes de Porcellio dilatafus, de systèmes hétérosomiques opposés

- homogamétie femelle (el hétérogamétie mâle) dans plusieurs populations continentales de la sous-espèce type, $P$. $d$. dilatatus : Poitiers, La Mérigotte (Vienne), Béziers (Hérault), île Sainte-Marguerite (Alpes-Maritimes) (Legrand et Juchault, 1974) et, en partie, la population de Neuvy-en-Dunois étudiée ici :

- hétérogamétie femelle (et homogamétie mâle) dans la population de l'île SaintHonorat de $P$. $d$. petiti et, en partie, la population de Neuvy-en-Dunois de la sousespèce type.

Une telle variation intraspécifique rappelle celle du Xiphophorus maculatus (Gordon, 1951, 1954 ; Kallman, 1965, 1970 ; Kallman et Atz, 1966). Néanmoins, chez ce poisson, une barrière géographique séparant deux populations a été invoquée pour expliquer l'évolution divergente du déterminisme sexuel hétérosomique. Ce processus semblait pouvoir s'appliquer au Porcellio dilatatus de l'île Saint-Honorat. La coexistence, à Neuvy-en-Dunois, de lignées différant par le système d'homohétérogamétie est peut-être le fait de l'homme, qui a pu réunir deux populations d'origines différentes. Néanmoins, un semblable mélange de lignées différant par le type d'homo-hétérogamétie a été observé dans la nature par Kallman (1965) pour les Xiphophorus maculatus de divers systèmes hydrographiques de l'Amérique centrale. Comme il est certain que l'origine de la lignée de Neuvy à hétérogamétie femelle n'est pas l'île de Saint-Honorat, dont la population correspond d'ailleurs à une sousespèce morphologiquement distincte, cette origine sera à rechercher dans la France continentale. L'inféodation de $P$. dilatatus aux grottes, dans l'ouest de la France (Legrand, 1956), peut néanmoins être favorable à un isolement génétique. Il en est peut-être de même de la vie dans les caves profondes des habitations, du moins dans une certaine mesure, comme semblent en témoigner les variations locales du taux des différents types d'intersexués (cf. ci-dessous).

On doit souligner, en conclusion de la présente étude, l'identité des valences sexuelles de la population de Saint-Honorat et de la lignée à hétérogamétie femelle de Neuvy, identité que l'on peut symboliser par la relation : (1) $X_{N}=X_{i}>Z_{N}=Z_{i}$, d'après les résultats des croisements du $\S$ VII.

L'hétérogamétie femelle de la population insulaire est concomitante de la présence d'un bactéroïde symbiote, transmis par les ovocytes et paraissant absent chez les mâles. La présente étude semble bien montrer que ce bactéroïde - s'il est res- 
ponsable de la fertilité de certains croisements entre individus de différentes populations - n'a probablement rien à voir avec la cause de l'hétérogamétie femelle, puisqu'il est absent dans la lignée homologue de Neuvy. Ce symbionte est donc de nature - et surtout d'effet — très différents de celui observé chez Armadillidium vulgare (Martin et al., 1973) et chez A. nasatum (Juchault et al., sous presse), qui transforme les mâles génétiques en néo-femelles. - La lignée de Neuvy-en-Dunois à homogamétie femelle (et hétérogamétie mâle) doit être très voisine de la population de Poitiers de la même sous-espèce. On peut symboliser cette similitude, d'après les croisements Neuvy-Poitiers, par les relations d'égalité ou d'épistasie des déterminants sexuels : (2) $Y_{N}=Y_{P}>X_{N}=X_{P}>Z_{N}$. Si l'on rapproche cette relation de la précédente (1), c'est donc une variabilité de la valence des déterminants sexuels mâles qui est à l'origine de la différence d'homo-hétérogamétie des populations étudiées. Une telle variation de la valence sexuelle rappelle les conclusions de Goldschmidt (1920) sur Lymantria dispar, à ceci près que les déterminants mâles et femelles étaient considérés comme variables avec les populations de cette espèce. Chez le Xiphophorus maculatus, Kallman (1965) conclut, par contre, à l'identité du chromosome $Y$ d'une lignée — ou population - à l'autre, ef à une variation portant sur le chromosome $X$ qui devient $W$ (épistatique sur $Y$ ) dans les lignées - ou populations — à hétérogamétie femelle.

La présente étude démontre que c'est la rencontre des lignées à homogamétie femelle et à hétérogamétie femelle qui est la cause de l'apparition périodique d'une arrhénogénie génétique de type $3 \hat{\delta} \delta / 1$ q dans la population de Neuvy-en-Dunois. Néanmoins, viennent s'ajouter d'autres formes de monogénie, découlant de l'inversion spontanée du sexe d'un pourcentage variable d'individus.

\section{Corrélation gènes-caractères sexuels.}

Nous avons montré (références in Legrand et Juchault, 1970) qu'une jeune femelle d'Oniscoïde peut être transformée en un néo-mâle fonctionnel, qui acquiert même une néo-glande androgène synthétisant l'hormone mâle. De même, un mâle génétique peut être transformé en néo-femelle si l'on introduit chez sa mère le bactéroïde féminisant $F$, qui inhibe la différenciation (post-embryonnaire) de la glande androgène (Juchault et Legrand, 1970). Ces éfudes expérimentales confirment, d'une part, qu'un mâle doit posséder tous les gènes responsables de la différenciation des caractères femelles, d'autre part, qu'une femelle doit également posséder tous les gènes responsables de la différenciation sexuelle mâle, y compris ceux qui contrôlent la synthèse de l'hormone androgène, mais qu'il lui manque le(ou les) gène(s) initiateur(s) de la différenciation de la glande androgène. Ces derniers - propres à un mâle génétique - pourraient exercer leur action au niveau d'un système de neurosécrétion contrôlant le fonctionnement de la glande androgène (Juchault et Legrand, 1978) puisque, chez un mâle féminisé par le bactéroïde $F$, c'est le système neurosécrétoire qui est initialement perturbé, et non la glande androgène. Chez un néomâle physiologique, un niveau de neurosécrétion du type mâle semble s'établir par effet feed back de l'hormone androgène allochtone, qui joue le rôle d'initiateur (Legrand, 1967).

L'obtention ici de néo-mâles physiologiques de génotype $X X\left(T_{5}\right)$ et $X Z$ (lignée à hétérogamétie femelle de Neuvy), confirmant ce qui a été obtenu, dans le 1 er cas, à partir de la lignée amphogène de Poitiers (Juchault et Legrand, 1964) et, dans le 2 e 
cas, à partir de la population de Saint-Honorat de Porcellio dilatatus petiti (Legrand et Juchault, 1974), montre que cette transformation s'obtient quelle que soit la constitution hétérochromosomique : homo ou hétérogamétique. Cependant, une nette différence dans la vitesse de la réponse s'observe dans ces différents cas pris 2 à 2 : l'inversion est 2 fois plus rapide avec une $q X Z$ qu'avec une $q X X$. Cependant, chez Armadillidium vulgare ( $q$ amphogène hétérogamétique), le temps de latence de l'inversion est long (mais on ne possède pas, dans ce cas, de référence vis-à-vis d'une $q$ homogamétique).

Partant de ces données physiologiques et des résultats de l'étude génétique précédente, on peut se représenter sous une forme relativement simple le mécanisme génétique fondamental de la détermination du sexe chez les Oniscoïdes. Quel que soit le système d'homo-hétérogamétie, on peut concevoir que le segment différentiel de l'hétérosome propre au mâle : $Y$ ou $Z$, porte le déterminant sexuel majeur $M$ contrôlanf la différenciation spontanée de la glande androgène. Les autres gènes sexuels, multiples ef communs aux 2 sexes, doivent être répartis sur les autosomes (et sur les segments pairs des hétérosomes). Le fait que, dans les croisements effectués ici entre lignée à homogamétie et lignée à hétérogamétie femelle, $Y$ et $Z$ soient interchangeables sans que la viabilité des descendants en soit altérée, montre que ces chromosomes sont très voisins. Néanmoins, associés à un même chromosome $X$, le résultat est très différent : mâle avec $Y$, femelle avec $X$. Un tel résultał met clairement en lumière la notion de «valence » du déterminant mâle. II peut s'agir d'une série d'allèles : $M_{Y}, M_{Z}$. Mais, quel est le rôle du chromosome $X$ ? Il n'est pas indispensable, même en face d'un $Y$, puisque les individus $Y Y$ sont des mâles viables (Legrand et Juchault, 1972b). L'hypothèse formulée par Gilchrist et Haldane (1947) chez Culex molestus - qui ne posséderait pas d'hétérochromosomes -, est que le sexe est contrôlé par un unique couple d'allèles : $\mathrm{Mm}=\hat{\delta}, \mathrm{mm}=$ Q , situé dans la même paire que le gène $w$ (œil blanc). Transposée chez Porcellio dilatafus, cette hypothèse revient à considérer que le segment différentiel de $X$ porte un allèle inactivant $M_{X}$, récessif par rapport à $M_{Y}$, mais dominant $M_{Z}$. Une autre hypothèse, envisagée pour différents animaux, consiste à proposer que, dans le couple $X Y, X$ introduit un effet modérateur qui a été rapporté par Goldschmidt (1965), Commoner (1964) et Mittwoch (1967) à l'hétérochromatine. Si, chez les Oniscoïdes, la structure des chromosomes n'a pas été étudiée, pas plus que l'effet sur le sexe des constitutions polysomiques $X Y Y$ ou $X X Y$, faute d'une visualisation des hétérochromosomes, les données qu'apporte l'étude précédente sont, d'une part, que la constitution femelle hétérogamétique permet une inversion expérimentale du sexe plus rapide, d'autre part que l'X semble identique dans toutes les populations étudiées (ici ef dans la publication de Legrand et Juchault, 1974).

Le premier point est favorable à l'hypothèse d'une localisation du ou des déterminants mâles majeurs sur $Z$ : la stimulation de ces gènes par l'hormone androgène allochtone conduirait à inverser la relation d'épistasie $(X>Z)$ (on peut également concevoir que l'hormone androgène atténue l'effet de $X$ ). Il est cependant à souligner que d'autres gènes sexuels, pairs, contrôlant la différenciation de la glande androgène doivent exister ailleurs que sur le segment différentiel de $Y$ ou $Z$, puisque les individus $X X$ peuvent être également inversés sexuellement : ceci appuie la conception d'un déterminisme polyfactoriel du sexe, comparable à celui de Winge (1934) 
chez Lebistes. Chez les femelles $X X$, la difficulté de l'inversion peut provenir d'une double dose de l'allèle inactivant ou du segment hétérochromatinien modérateur.

Le deuxième point stipule une stabilité génétique de $X$, vis-à-vis d'une variabilité de son complément, $Y$ ou $Z$. Ceci pourrait orienter une certaine préférence vers l'hypothèse qu'X porfe un système modérateur ef non un allèle de $M$.

3. Facteurs de l'inversion spontanée du sexe.

II existe dans les populations de Porcellio dilatatus, différentes anomalies de la différenciation sexuelle et de la sex-ratio susceptibles d'être rapportées à l'action de facteurs épigénétiques.

a) On observe, d'une population à l'autre une grande variabilité des types d'intersexualité, ou de leurs taux respectifs, déjà notée précédemment (Legrand, 1948), (Juchault, 1966).

- A Poitiers, il a été observé à la fois :

- des femelles à apophyse génitale mâle ( $q$ ap), qui sont génétiquement des mâles, inversés par un facteur féminisant labile, $f$,

- des mâles à ouvertures génitales femelles (ơog), (également présents dans différentes populations d'Armadillidium vulgare, où cet état a été rapporté à une inversion sexuelle des femelles par un facteur, $m$, probablement épigénétique),

- des femelles à pseudohermaphrodisme masculin externe de type B (Juchault, 1966).

- A Saint-Honorat (résultats inédits), on observe à la fois des ô og - qui sont des néo-mâles - et des femelles à tractus génital mâle (sans doute voisines des $q$ ap). - A Corbeil (Essonne), Roscoff (Finistère), Chinon (Indre et Loire), ont été trouvés les mêmes types d'intersexualité qu'à Poitiers mais avec une fréquence supérieure. - A Neuvy-en-Dunois, n'ont été observés que les deux types (A et $B$ ) de pseudohermaphrodisme masculin externe, rapportés à un facteur épigénétique infectieux (virus ?) ; par contre, les $q$ ap sont inconnues et les 0 og rarissimes (un seul observé sur les 3 récoltes).

b) Quelle est - chez Porcellio dilatatus - la relation entre intersexualité ef monogénie découlant de l'inversion du sexe?

$b_{1}$. - Facteurs féminisants ef inversion sexuelle des mâles en néo-femelles normales ou intersexuées.

Jusqu'ici, seules certaines populations de $P$. dilatatus (La Mérigotte, Chinon) (Legrand et Juchault, 1972b) ont offert des phénomènes sporadiques de monogénie, liés à certaines formes d'intersexualité, mais découlant simplement de l'inversion sexuelle de rares individus mâles : c'est le cas des $q$ ap qui sont des néo-femelles $X Y$ : croisées avec des mâles normaux, elles donnent une arrhénogénie du type $3 \hat{\delta} \sigma^{\wedge} / 1$ q et, en $F_{2}$, par suite de la réalisation, en $F_{1}$, de mâles $Y Y$, apparaît une arrhénogénie pure dans la proportion $1(\mathrm{Ar}) / 2(\mathrm{~A})$. Il s'agit donc d'une monogénie génétique ef tout rentre dans l'ordre à la génération suivante, bien que la réapparition d'inversés puisse relancer le phénomène.

- A Neuvy-en-Dunois, l'apparition - beaucoup plus fréquente - de néofemelles $Z_{N} Z_{N}$ est à l'origine de deux types de monogénie : d'une part, une arrhé- 
nogénie pure - ou presque pure - génétique, susceptible de se perpétuer par suite de la réapparition de néo-femelles dans la descendance, d'autre part, une thélygénie partielle (37 à 40 p. 100 de mâles), transmissible et susceptible d'être sélectionnée (cf. III.6b). Il est à souligner que l'arrhénogénie pure n'a été observée que dans la descendance de ces formes de thélygénie partielle. Ces dernières coexistent avec des descendances amphogènes dont elles prennent la place; ceci permet de reconstituer la filiation :

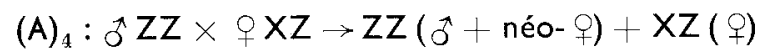

et : $\widehat{\jmath} \mathbf{Z Z} \times$ néo- $q \mathbf{Z Z} \rightarrow \mathbf{Z Z}(\hat{\jmath}+$ néo- $q)$.

Si l'on fait le total des descendances offrant une arrhénogénie pure - ou forte apparues dans les filiations à tendance $(T)$ étudiées, on en compte $16 / 130$, soit 12,3 p. 100. Ce taux correspond donc approximativement à celui des néo-femelles $Z Z$ existant dans ces filiations. Il suffit ainsi à expliquer cette thélygénie partielle puisqu'il rend compte des 60 à 63 p. 100 de femelles. Il est à souligner que la femelle joue un rôle essentiel dans la transmission de la thélygénie partielle, ainsi qu'en témoignent les résultats exposés en VII $2 b_{3^{-4}}$. Ce fait est plutôt en faveur d'une nature épigénétique du facteur d'inversion du sexe, ce qui le rapproche du facteur féminisant, $f$, d'Armadillidium vulgare (Legrand ef Juchault, 1972a).

- C'est peut-être le même facteur qui est responsable de l'excès significatif de femelles par rapport à la proportion $3 \hat{\delta} \delta / 1$ q, observé dans beaucoup de descendances (Ar), où coexistent précisément des femelles $X Z$ et des mâles $Z Z$.

- Y a-t-il possibilité d'inversion des mâles hétérogamétiques $(X Y)$, comme dans d'autres populations? Il n'a pas été observé, à Neuvy, de $q$ ap qui accompagnent ce type d'inversion. Par ailleurs, on devrait observer, si cela existait, l'apparition, à la génération suivante, de l'arrhénogénie de type $3 \delta \delta / 1$ 으. indiscernable, à ce stade, de celle qui découle de l'hybridation des lignées à hétéro- ef à homogamétie femelle, puis, ultérieurement, de l'arrhénogénie pure due aux mâles YY. Or, il n'a jamais

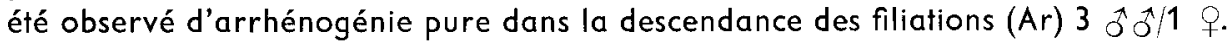

- Nous avons vu, par contre, que les mâles $X_{N} Y_{N}$ de la lignée $T s_{5}$ de Neuvy engendraient moins de mâles, quelle que soit la femelle avec laquelle ils sont croisés. Il semble que ce processus n'a rien à voir avec une inversion de ces mâles en néofemelles. Ceci serait en effef à l'encontre du résultat maintes fois constaté (Legrand et Juchault, 1974), à savoir que le croisement avec un individu d'une autre population modifie la transmission des facteurs d'inversion du sexe, ce qui n'a pas été observé ici, dans le croisement d'un mâle $T s_{5}$ avec une femelle poitevine.

L'explication de la déficience de mâles pourrait être une moindre fertilité des spermatozoïdes $A Y_{N}$ vis-à-vis de celle des spermatozoïdes $A X_{N}$.

$b_{2}$. - Facteurs masculinisants.

L'apparition sporadique de néo-mâles spontanés $X_{N} Z_{N}$ - qui conduisent, par croisement avec des femelles sœurs, à une thélygénie du type $1 \partial^{*} / 3$ q $q$ et, à la $F_{2}$, à une thélygénie pure, par suite de la présence de femelles $X X$ (en nombre moitié de celui des femelles $X Z$ ) - témoigne de l'existence d'un facteur masculinisant suffisamment puissant pour inverser le sexe, mais trop labile ef rare pour induire une monogénie durable. On doit néanmoins souligner qu'un tel facteur permet une transition entre hétérogamétie femelle et homogamétie femelle puisque les individus $X X$ 
nés en $F_{1}$ du croisement néo- $\hat{\sigma} X Z \times$ q $X Z$ paraissent identiques aux femelles homogamétiques du type $T s_{5}$ ou poitevin. Ces néo-mâles spontanés sont à rapprocher de ceux éfudiés par Tinturier-Hamelin (1963) chez Idotea balthica, particulièrement fréquents dans certaines populations ( $I . b$. tricuspidata de la Manche et de l'Atlantique).

En ce qui concerne le $P$. dilatatus de Neuvy, il est possible que le mâle soit pour quelque chose dans la transmission du facteur masculinisant (cf. III.6a). Ce facteur est-il de nature épigénétique ou génétique? Est-il identique au facteur responsable de l'intersexualité des femelles (type A du pseudohermaphrodisme masculin externe) ? Ce dernier est épigénétique puisque susceptible d'être introduit chez une femelle normale par implantation d'un fragment quelconque de tissu de femelle intersexuée de type A (Juchault et Legrand, 1970, 1975). La disparition en élevage de cette forme d'intersexualité dès la $F_{2}$ de la descendance des femelles de la nature, tendrait à prouver qu'il n'est pas responsable de l'apparition de néo-mâles. Cependant, l'abondance des femelles intersexuées de type $B$ dans la filiation Neuvy pure (ex. $\left.F_{3} B i l\right)$, pose le problème d'une relation entre facteurs responsables des 2 types ( $A$ et $B$ ) d'intersexualité. S'il s'agit de deux formes voisines d'un même microorganisme (virus ?), on ne peut plus parler d'une labilité de ce facteur et la question demeure de son identité arec celui qui est cause de l'inversion du sexe femelle. Remarquons néanmoins que l'intersexualité du type $B$ est très tardive, ce qui ne convient pas à l'inversion du sexe, obligatoirement très précoce. Il peut s'agir cependant, dans ce dernier cas, d'une forme plus active ou d'une abondance particulière du facteur dans les œufs fécondés. Le facteur de l'intersexualité de type A étant thermolabile (il disparaît à $27^{\circ} \mathrm{C}$ ) (Juchault ef Legrand, 1975), l'étude de l'action de la température sur le taux des néomâles serait un moyen de comparer les deux types de facteurs. Elle n'a pu être menée ici, faute d'un nombre suffisant de descendances offrant ces néo-mâles et par suite de la rareté de ces derniers.

Qu'il soit génétique ou épigénétique, on peut concevoir que le facteur d'inversion du sexe exerce une action synergique de celle du, ou des déterminants mâles contenus dans $Z_{N}$, et qu'il inhibe l'épistasie de $X$ sur $Z$. Un tel processus appuie l'idée que le sexe est bien la résultante d'une balance entre facteurs sexuels antagonistes. Il est d'ailleurs remarquable - comme cela a été observé sur d'autres matériels, et notamment Idotea balthica (Tinturier-Hamelin, 1963) - que le croisement d'un néomâle et d'une néo-femelle rétablit l'amphogénie (III.7), ce qui implique le rétablissement, dans les descendants des 2 sexes, d'une balance génique normale.

c) Mécanisme de l'allélogénie.

L'allélogénie, qui correspond - dans la descendance d'un même couple à une succession de portées différant de façon significative par la proportion des sexes, est un phénomène relativement rare : 25 cas ont été observés sur plusieurs milliers de croisements. Il est intervenu dans presque tous les types de descendances : arrhénogénie de type $3 \delta \delta / 1$ i ( 3 cas), exemple, dans la $F_{3}$ d'un croisement entre

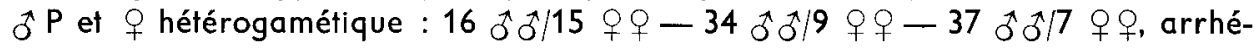

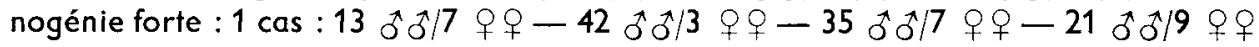

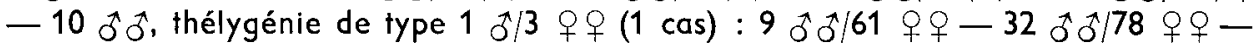
$13 \delta \delta^{\top} / 43$ Q $Q$, tendance thélygène $(3 \mathrm{cas})$, exemple, dans la $F_{2}$ d'un croisement à

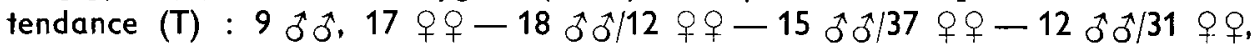


mais surtout, amphogénie (14 cas). II est également réapparu en $F_{2}$ de descendances allélogènes ( 3 cas), mais ce type de filiation n'a pas été étudié de façon exhaustive.

En ce qui concerne l'arrhénogénie forte ef la tendance thélygène, la variation significative de la sex-ratio peut être rapportée à celle du taux des néo-femelles. Un processus analogue - mais qui n'a pas été comptabilisé ici - correspond à l'apparition irrégulière de néo-mâles dans les différentes portées d'une descendance qui aurait dû être thélygène pure. On peut donc concevoir, dans un certain nombre de cas, que l'allélogénie a pour base une variation dans le temps du taux des inversés sexuels. Il est à souligner que, si la photopériode offre une grande importance en matière de synchronisme de la reproduction chez les Oniscoïdes (Mocquard ef al., 1976, 1978), ce facteur - le seul qui varie dans nos élevages, soumis à une lumière naturelle atténuée - ne paraît pas jouer ici car on ne peut rapporter l'augmentation - ou la diminution - des inversés sexuels à une saison particulière. Il faut donc incriminer un facteur endogène dont la variation offre un caractère aléatoire.

Un tel processus est favorable à l'hypothèse de l'intervention de facteurs épigénétiques dont le taux dans chacun des ovocytes d'une ponte - ou des spermatozoïdes d'une même poussée spermatogénétique - serait très variable, mais essentiel pour l'évolution sexuelle des embryons. Il a d'ailleurs été prouvé que de tels facteurs antagonistes, $m$ ef $F$ peuvent coexister dans un zygote et qu'ils conditionnent l'état intersexué des individus de la lignée thélygène $T_{\underline{F}}$ d'Armadillidium vulgare (Legrand et al., 1974).

Qu'en est-il au sujel des autres descendances allélogènes? La majorité d'entre elles relève sans doute des cas extrêmes d'une variation gaussienne de la proportion des sexes centrée sur le rapport 1/1. Il en est probablement de même pour les autres

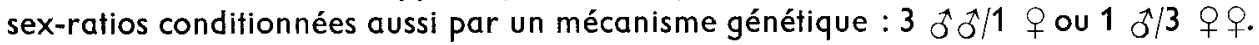
Il est d'ailleurs à souligner que la tendance thélygène de certaines descendances associées à une forte majorité d'amphogènes n'a pas présenté un caractère héréditaire (exemple en $\mathrm{F}_{6} \mathrm{Ar}_{7}$ ) (cf. fig. 2).

Il est très possible, en fait, que les deux types de variations aléatoires : génétique et épigénétique, se combinent, sauf lorsqu'il s'agit de descendances thélygènes et arrhénogènes «pures », où des facteurs épigénétiques pourraient être seuls en cause dans l'allélogénie.

Reçu en janvier 1979.
Accepté en mars 1979.

\section{Références}

ARTAULT J. C., 1977. Contribution à l'étude des garnitures chromosomiques chez quelques Crustacés Isopodes. Th. 3e Cycle, Poitiers, no 665, 63 pp., 7 pl.

BACCI G., 1965. Sex determination. Pergamon Press.

COMMONER B., 1964. Roles of desoxyribonucleic acid inheritance. Nature, 202, 960-968.

GILCHRIST B. M., HALDANE J. B. S., 1947. Sex linkage and sex determination in a mosquito, Culex molestus. Hereditas, 33, 175-190.

GOLDSCHMIDT R., 1920. Untersuchungen über Intersexualität. I. Z. indukt. Abstomm. u. Vererb Lehre, 23, 1-199. 
GOLDSCHMIDT R., 1938. Physiological genetics. Mc Graw-Hill New York.

GOLDSCHMIDT R., 1955. Theorefical genetics. Univ. of California Press, Berkeley.

GORDON M., 1951. Genetics of Platypoecilus maculatus. V. Heterogametic sex-determining mechanism in females of a domesticated stock originally from British Honduras. Zoologica, 36, 127-134.

GORDON M., 1954. Two opposing sex-determining mecanisms, one XX-XY, the other WY-YY, in different natural populations of the platyfish Xiphophorus moculatus. Caryologia, 6, Suppl., 960-964.

JUCHA ULT P., 1966. Contribution à l'élude de la différenciation sexuelle mâle chez les Crustacés Isopodes. Thèse, Poitiers, 148 pp., 11 pl.

JUCHAULT P., LEGRAND J. J., 1964. Démonstration de l'homogamétie femelle par croisement de deux femelles génétiques chez les Oniscoïdes Porcellio dilatatus et Helleria brevicornis. C. R. Acad. Sci. Paris, 258, 2685-2686.

JUCHAULT P., LEGRAND J. J., 1970. Intersexualité et monogénie chez les Crustacés Isopodes terrestres : induction de la thélygénie chez Armadillidium vulgare; facteurs contrôlant le pseudo-hermaphrodisme masculin externe chez Porcellio dilatatus. Ann. Endocrinol. Paris, 31, 525-530.

JUCHAULT P., LEGRAND J. J., 1975. Modalités de la transmission héréditaire du facteur épigénétique responsable de l'intersexualité masculinisante chez le Crustacé Oniscoïde Porcellio dilatafus Brandt. Bull. Soc. Zool. Fr., 100, 467-476.

JUCHAULT P., LEGRAND J. J., 1978. Etude du fonctionnement de la glande androgène dans le cas d'implantations croisées entre deux espèces de crustacés isopodes terrestres, Porcellio dilatatus Brandt et Armadillidium vulgare Latreille : notion de spécificité de l'hormone androgène et des neurohormones impliquées dans le contrôle de la fonction androgène. Gen. Compar. Endocr., 36, 175-186.

JUCHAULT P., LEGRAND J. J., 1979. Analyse génétique et physiologique de la détermination du sexe dans une population du Crustacé Isopode Oniscoïde Armadillidium nasatum BuddeLund. Arch. Zool. exp. Gén., 120, 25-43.

KALLMAN K. D., 1965. Genetics and geography of sex determination in the poeciliid fish, Xiphophorus maculatus. Zoologica, 50, 151-190.

KALLMAN K. D., ATZ J. W., 1966. Gene and chromosome homology in fishes of the genus Xiphophorus. Zoologica, 51, 107-134.

KALLMAN K. D., 1970. Sex determination and the restriction of sex-linked pigment patterns to the $X$ and $Y$ chromosomes in populations of a poeciliid fish, Xiphophorus maculatus, from the Belize and Sibun rivers of British Honduras. Zoologica, 55, 1-16.

LEGRAND J. J., 1947. Cas d'inversion du sexe chez Porcellio dilatatus Brandt. C. R. Acad. Sci. Paris, 224, $1029-1031$.

LEGRAND J. J., 1948. Sexualité des Isopodes terrestres. Travoux du XIIIe Congr. Int. Zool. Paris.

LEGRAND J. J., 1956. Contribution à l'étude de la faune cavernicole de l'ouest de la France. I. Grottes visitées en 1954 et 1955. Etude des Crustacés Isopodes terrestres récoltés. Notes Biospeolog., XI, 23-42.

LEGRAND J. J., 1962. Contribution à l'étude de la génétique de la pigmentation chez les Oniscoīdes : étude de six gènes et de leurs corrélations chez Porcellio dilatatus Brandt. 87e Congr. Soc. savantes, $1143-1151$.

LEGRAND J. J., 1967. Contribution à l'étude du contrôle génétique et humoral de l'inversion du sexe chez les Crustacés isopodes. Notion de balance génique sexuelle. Ann. Biol., VI, 5-6, 241-258.

LEGRAND J. J., 1977. Déterminisme génétique et épigénétique du sexe chez les Crustacés Isopodes terrestres; corrélation entre monogénie et intersexualité. Bull. Soc. Sci. nat. Tunisie, 12, 3-12.

LEGRAND J. J., JUCHAULT P., 1970. Le contrôle humoral de la sexualité chez les Crustacés isopodes gonochoriques. Cours et documents de Biologie. Gordon and Breach.

LEGRAND J. J., JUCHAULT P., 1972a. Mise en évidence dans une population d'Armadillidium vulgare Latr. (Crustacé Isopode Oniscoïde) de deux types de lignées arrhénogènes en relation avec des facteurs épigénétiques à effet respectivement masculinisant et féminisant. $C$. $R$. Acad. Sci. Paris, 274, 1554-1557. 
LEGRAND J. J., JUCHAULT P., 1972b. Contribution à l'analyse du contrôle de l'hermaphrodisme protandrique rudimentaire de l'Oniscoïde Porcellio dilatatus et de la monogénie occasionnelle liée à cette forme d'intersexualité. C. R. Acad. Sci. Paris, 274, 2232-2235.

LEGRAND J. J., JUCHAULT P., 1974. Contribution à l'étude de la génétique du sexe chez les Oniscoïdes. Analyse des croisements entre deux sous-espèces de Porcellio dilatatus Brandt, l'une à hétérogamétie mâle, l'autre à hétérogamétie femelle. Arch. Zool. exp. Gen., 115, 441-488.

LEGRAND J. J., JUCHAULT P., ARTAULT J. C., MOCQUARD J. P., PICAUD J. L., 1976. Le statut systématique de la «forme » petiti Vandel de Porcellio dilatatus Brandt récoltée à l'île SaintHonorat (Alpes Maritimes). Critères morphologiques, génétiques et physiologiques. Bull. Soc. Zool. Fr., 99, 461-471.

LEGRAND J. J., JUCHAULT P., MOCQUARD J. P., 1974. Analyse préliminaire du mécanisme de l'intersexualité féminisante chez le Crustacé Armadillidium vulgare Latr. (Isopode, Oniscoïde). C. R. Acad. Sci. Paris, 278, 2979-2982.

LEGRAND J. J., LEGRAND H., 1947. L'intersexualité chez l'Oniscoïde Porcellio dilatatus Brandt. C. R. Acad. Sci. Paris, 224, 353-355.

LEGRAND J. J., MARTIN G., ARTAULT J. C., 1979. Corrélation entre la présence d'un symbionte bactérien dans les ovocytes de Porcellio dilatafus petiti, et la stérilité du croisement: $P$. $d$. petiti $3 \times$ P. d. dilatatus ?. Bull. Soc. Sci. nat. Tunisie (sous presse).

MARTIN G., JUCHAULT P., LEGRAND J. J., 1973. Mise en évidence d'un micro-organisme intracytoplasmique symbionte de l'Oniscoïde Armadillidium vulgare Latr. dont la présence accompagne l'intersexualité ou la féminisation totale des mâles génétiques de la lignée thélygène. C. R. Acad. Sci. Paris, 276, 2313-2316.

MITTWOCH V., 1967. Sex chromosomes. Acad. Press, New York, London.

MOCQUARD J. P., BESSE G., JUCHAULT P., LEGRAND J. J., MARTIN G., MAISSIAT J., PICAUD J. L., 1976. Durée de la période de reproduction chez la femelle de l'Oniscoïde Parcellio dilatatus Brandt suivant les conditions d'élevage : température, photopériode et groupement. Vie et Milieu, 26, 51-76.

MOCQUARD J. P., BESSE G., JUCHAULT P., LEGRAND J. J., MARTIN G., MAISSIAT J., PICAUD J. L., 1978. Action de la température et de la photopériode sur l'induction des mues parturielles des femelles de Porcellio dilatatus Brandt (Crustacé Isopode Oniscoïde). Arch. Zool. exp. Gén., 119, 409-432.

TINTURIER-HAMELIN E., 1963. Polychromatisme et détermination génétique du sexe chez l'espèce polytypique Idotea balthica (Pallas) (Isopode Valvifère). Cah. Biol. mar., 4, 473-591.

VANDEL A., 1962. Faune de France. Isopodes ferrestres. $2^{\mathrm{e}}$ partie. Paul Lechevalier, Paris, 514 pp.

WINGE O., 1934. The experimental alteration of sex chromosomes into autosomes and vice versa, as illustrated by Lebistes. C. R. Trav. Lab. Car/sberg, 21, 1-49. 